\title{
35. DETAILED STRATIGRAPHIC CORRELATION OF THE NEOGENE SEDIMENTARY SEQUENCES ON THE ONTONG JAVA PLATEAU BY WELL LOGGING: ODP SITES 803, 805, 806, 807, AND DSDP SITE 586 ${ }^{1}$
}

\author{
Mitchell Lyle, ${ }^{2}$ Roy H. Wilkens, ${ }^{3}$ and Larry A. Mayer ${ }^{4}$
}

\begin{abstract}
We used well logs, in some cases combined with shipboard physical properties measurements to make more complete profiles and to correlate between sites on the Ontong Java Plateau. By comparing sediment bulk density, velocity, and resistivity logs from adjacent holes at the same site, we showed that even subtle features of the well logs are reproducible and are caused by variations in sedimentation. With only minor amounts of biostratigraphic information, we could readily correlate these sedimentary features across the entire top of the Ontong Java Plateau, demonstrating that for most of the Neogene the top of the plateau is a single sedimentary province. We found it more difficult, but still possible, to correlate in detail sites from the top of the plateau to those drilled on the flanks. The pattern of sedimentation rate variation down the flank of the plateau cannot be interpreted as simply controlled by dissolution. Site 805 , in particular, oscillates between accumulating sediment at roughly the same rate as cores on top of the Ontong Java Plateau, and accumulating sediment as slowly as Site $803,200 \mathrm{~m}$ deeper in the water column. These oscillations do not match earlier reconstructions of central Pacific carbonate compensation depth variations.
\end{abstract}

\section{INTRODUCTION}

The sediments of the Ontong Java Plateau potentially contain a detailed record of the Neogene evolution of the western equatorial Pacific Ocean, provided that one can separate local variations in sedimentation from regional features related to paleoceanographic change. In this paper, we will examine the changes in sedimentation at drill sites across the top of the Ontong Java Plateau and down its eastern flank (Fig. 1) to search for regionally coherent patterns of sedimentation and to discover whether it is possible to observe and refine the Neogene changes in the carbonate compensation depth (CCD) reported for the central equatorial Pacific Ocean (Berger, 1973; van Andel et al., 1975). Because all of the sites are from above the actual CCD, we hoped to monitor $\mathrm{CCD}$ changes by the dissolution pattern at sublysocline drill sites. By comparing sediment accumulation for equivalent intervals, we hoped to monitor dissolution down the flank of the plateau.

Stratigraphic correlation is the basis for any paleoceanographic study involving more than one sediment core or Ocean Drilling Program (ODP) drill site. For reasonably complete ODP sections, the stratigraphic control of choice is the oxygen isotopic record of benthic foraminifers, because it is well known that this signal is synchronous within about a $1000 \mathrm{yr}$ throughout the oceans and because the measurement of oxygen isotopes on foraminifers has become routine (Shackleton et al., 1984; Imbrie et al., 1984; Martinson et al., 1987). This correlation method has its drawbacks, however, in older sediments and for thick sedimentary sequences. First of all, a complete stratigraphic record is dependent upon core recovery. When the sediments become lithified and core recovery drops, difficulties quickly arise in assigning specific oxygen isotope excursions to the master stratigraphic record. For thick sedimentary sequences, too, it

\footnotetext{
'Berger, W.H., Kroenke, L.W., Mayer, L.A., et al., 1993. Proc. ODP, Sci. Results, 130: College Station, TX (Ocean Drilling Program).

${ }^{2}$ Borehole Research Group, Lamont-Doherty Geological Observatory, Columbia University, Palisades, NY 10964, U.S.A. (present address: 5840 Collister Drive, Boise, ID 83703, U.S.A.).

${ }^{3}$ Institute of Geophysics, School of Ocean and Earth Science and Technology, University of Hawaii, 2525 Correa Road, Honolulu, HI 96822, U.S.A.

${ }^{4}$ Ocean Mapping Group, Department of Surveying and Engineering, University of New Brunswick, P.O. Box 4400, Fredericton, New Brunswick, E3B 5A3 Canada.
}

takes too long and costs too much to measure oxygen isotopes in the detail needed for stratigraphic purposes.

For these reasons, we decided to investigate whether well logs could be used for stratigraphic control for high-resolution paleoceanographic studies. Well logs have the advantage that the data is continuously recorded and are available at sea where they potentially can be used by shipboard scientists during drilling operations and in the initial interpretation of the sediment section. The data are of the best quality in the lithified sections where core recovery begins to drop and where continuous composite sections cannot be reconstructed from recovered core. And, by recording a variety of physical and chemical properties of the sediments, the ODP well logging program provides multiple potential stratigraphic tools.

The sediments of the Ontong Java Plateau provide a good test of the use of well logs for high-resolution stratigraphy. The Neogene sediment column everywhere on the plateau is a monotonous sequence of pelagic carbonate oozes (Kroenke, Berger, Janecek, et al., 1991). Only subtle variations in physical properties were noted in the cores, except for diagenetic cementation. Although no distinct sedimentary layers can be used for correlation, the biostratigraphy is excellent (Kroenke, Berger, Janecek, et al., 1991). Sedimentary layers on the plateau should have major lateral extent because the Ontong Java Plateau has always been pelagic (Resig et al., 1976) and because the plateau has always been under the open ocean. The continuity of sedimentary layers is indicated by seismic reflection records across the plateau (Mayer et al., 1991). Acoustic reflectors can be correlated easily from one side of the Ontong Java Plateau to the other. Well logs, with their better resolution than seismic reflection, should prove to be even more useful for stratigraphic correlation.

Our strategy, in this paper, is to first demonstrate the reproducibility of well log data by comparing logs of duplicate sections in Holes $807 \mathrm{~A}$ and $807 \mathrm{C}$. Then, when we have established the precision with which well $\log$ data can be recorded, we will use an inverse correlation technique (Martinson et al., 1987) to map each site to the standard Neogene stratigraphic section, represented by the section from Hole $806 \mathrm{~B}$. In addition, the Paleogene/Neogene sections recovered in Sites 807 and 803 will be correlated to each other. After we have correlated the sections, we will discuss the paleoceanographic implications and use a crude time scale to investigate the periodicities in our records.

All well logs used in this paper were recorded by Schlumberger, using their standard logging tools. A description of the tools used and 




Figure 1. Bathymetry of the Ontong Java Plateau showing the locations of the drill sites on ODP Leg 130 (803-807) and DSDP Leg 89 Site 586.

the operational conditions in each hole are given in Kroenke, Berger, Janecek, et al. (1991). In general, hole conditions and the depth at which we set pipe for the logging runs prevented the use of logging data for the correlation of Pliocene-Pleistocene sections. Good correlations from the logs begin before about $5 \mathrm{Ma}$ (latest Miocene) and continue back in time until diagenetic cherts become common in the section, in the Eocene.

\section{CORRELATION OF HOLES 807A AND 807C}

The physical properties variations we measured on the Ontong Java Plateau with well logs are subtle, and for this reason we wanted to confirm their reproducibility. Figure 2 illustrates the level of variation for the shallow focused resistivity $\log$ (SFLU), played back at a standard scale used in the oil industry. Note how the entire 


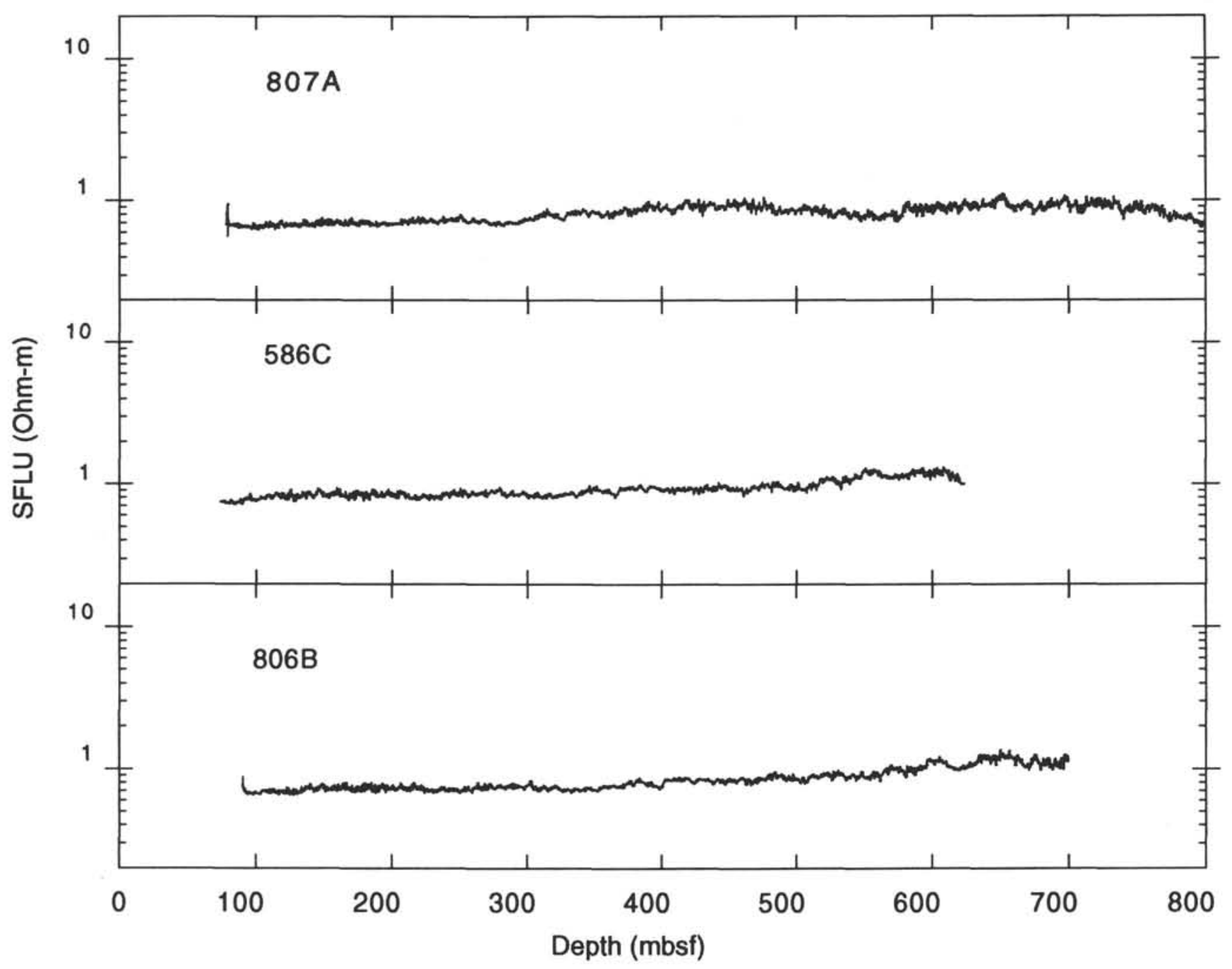

Figure 2. The shallow-focused resistivity logs for the three sites on top of the Ontong Java Plateau at a scale typically used to display ODP logs. The figure illustrates how small are the variations in the Neogene ooze and chalk sequence. For logs to be useful for paleoceanography, even these small variations must be reproducible.

Neogene section has only minor resistivity changes (the Miocene/ Oligocene boundary is at about $740 \mathrm{mbsf}$ in Hole 806B, at $590 \mathrm{mbsf}$ in Hole 807A, and would be at about 700 mbsf at Site 586). For such variations to be useful for stratigraphy, they must be reproducible.

Hole 807A is the pilot hole drilled at Site 807, in a water depth of $2803.8 \mathrm{~m}$. It was with the advanced hydraulic piston corer (APC) and the extended core barrel (XCB) to 822.9 mbsf and logged with the standard logging suite from $820 \mathrm{mbsf}$ to the end of pipe at $85.6 \mathrm{mbsf}$. Hole $807 \mathrm{C}$ was drilled about $100 \mathrm{~m}$ north of Hole $807 \mathrm{~A}$, for a reentry hole to basement and was cased to $350 \mathrm{mbsf}$. Nevertheless, we were able to $\log$ in Hole $807 \mathrm{C}$ between 350 and 820 mbsf a section equivalent to the one in Hole $807 \mathrm{~A}$. The two holes provide a means to test the level to which hole conditions and tool response limit our ability to correlate between holes by means of logs.

With only minor depth shifting, discussed in more detail later, we can get excellent reproducibility between the logs from the two holes (Fig. 3). The worst disagreement occurs with the shallow focused resistivity log in the interval above $520 \mathrm{mbsf}$; this is caused by uncorrected borehole size effects in the log data. These types of effects shift the baseline resistivity values but still leave the high-frequency variations intact. We can examine the scale of variation by comparing the Hole $807 \mathrm{~A}$ logs to the Hole $807 \mathrm{C}$ logs in a typical interval, between 550 and 600 mbsf. In each case, although slight baseline offsets may be present, we find that the residual standard deviations are $<0.02 \mathrm{ohm}-\mathrm{m}$ for SFLU, $<0.02 \mathrm{~g} / \mathrm{cm}^{3}$ for wet-bulk density, and $<0.02 \mathrm{~km} / \mathrm{s}$ for sonic velocity.

\section{CORRELATION OF ALL ONTONG JAVA PLATEAU SITES}

We used the SFLU shallow resistivity log to correlate the drill sites on top of the Ontong Java Plateau $(806,807$, and 586) because this $\log$ has the highest frequency response of any of the logs we recorded on each hole. For sites down the margin of the plateau (803 and 805), we found that the bulk density logs are significantly more coherent with logs from sites on top of the Ontong Java Plateau than the SFLU, as we will discuss later. We used an inverse signal correlation technique, described in Martinson et al. (1982) and available as a computer package (CORPAC), to correlate among the drill sites. Briefly, the technique involves comparing a "distorted" time or depth series to a reference record and recovering a mapping function that makes the two similar. The aim of the inverse technique is to maximize 


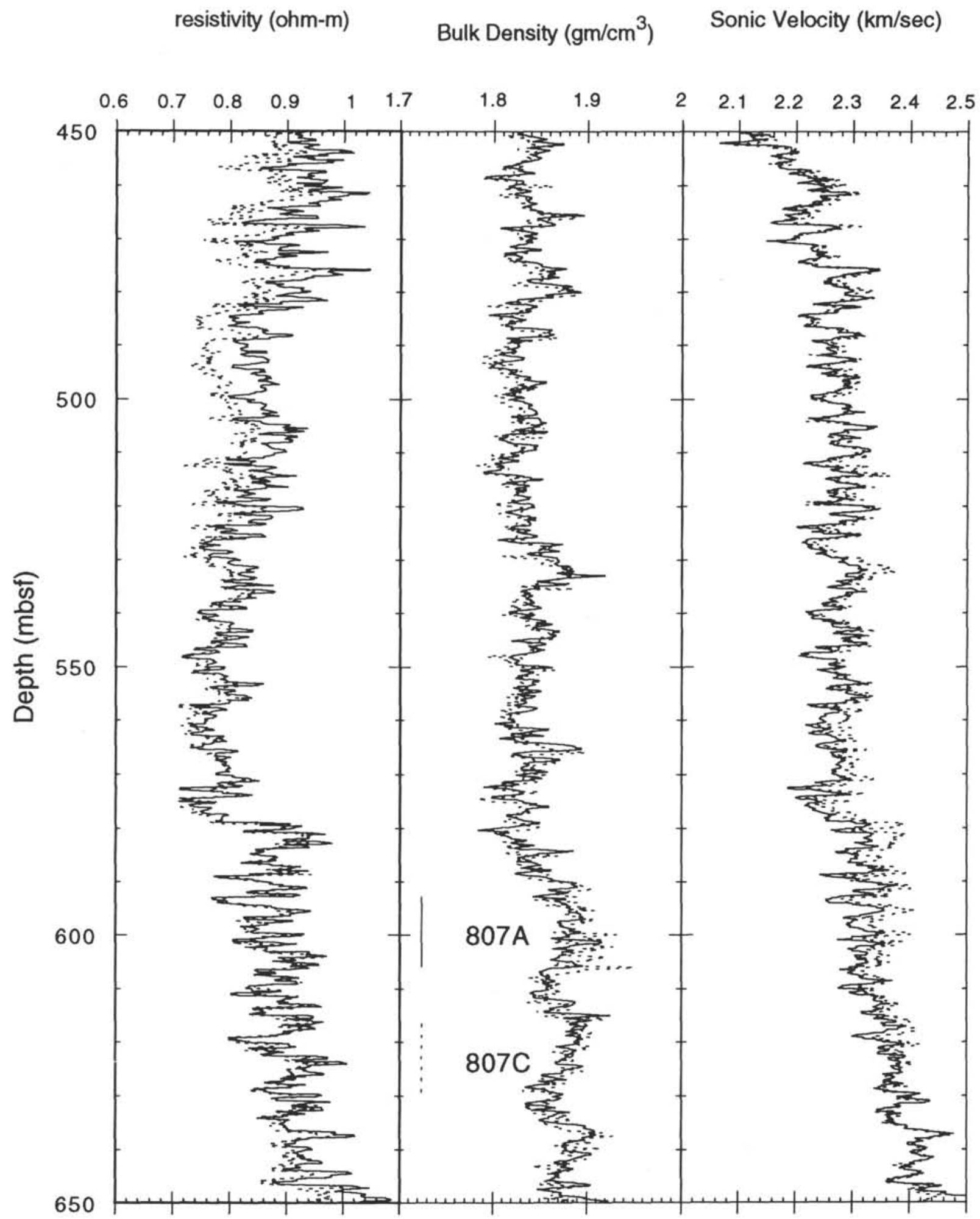

Figure 3. A comparison of logging data between Holes $807 \mathrm{~A}$ and $807 \mathrm{C}, 100$ yards apart. The data were depth shifted by the inversion technique described in the paper to remove the effects of minor sedimentation rate variations between the two holes. Sonic velocity $(\mathrm{km} / \mathrm{sec})$ is from the near-spaced sensors; wet bulk density in $\mathrm{gm} / \mathrm{cm}^{3}$; resistivity, from the shallow focused resistivity log (ohm-m). 
coherence between the two normalized time series by assuming that the mapping function is a combination of linear and harmonic distortions. It iterates from an initial guess along the gradient of maximum coherence increase per change in the harmonic coefficients until coherence is maximized. The computer package is also interactive. We used a few biostratigraphic tie-points between holes as the initial guess for the correlation and graphically compared the resulting computer-generated correlation. We used this comparison to redefine the tie-points and repeat the process. Afterward, we compared this correlation to the other independent biostratigraphic information.

\section{The Ontong Java Plateau Top: Sites 806, 586, and 807}

The three sites on top of the Ontong Java Plateau-Site 806 $\left(00^{\circ} 19.11^{\prime} \mathrm{N}, 159^{\circ} 21.66^{\prime} \mathrm{E}, 2520 \mathrm{~m}\right.$ water depth), DSDP Site 586 $\left(00^{\circ} 29.84^{\prime} \mathrm{S}, 158^{\circ} 29.89^{\prime} \mathrm{E}, 2208 \mathrm{~m}\right.$ water depth), and Site 807 $\left(3^{\circ} 36.36^{\prime} \mathrm{N}, 156^{\circ} 37.5^{\prime} \mathrm{E}, 2804 \mathrm{~m}\right.$ water depth) - demonstrate the facility with which holes can be correlated under ideal conditions. The quality of the correlation also shows that the sediments at each of the sites on top of the Ontong Java Plateau contain a record of regional paleoceanographic events and not local sedimentary accidents.

At each site, we used the drill string "bottom felt" data to convert from meters below the rig floor to meters below seafloor. We subtracted $2531.0 \mathrm{~m}$ from the total wire out on the logging runs for Hole $806 \mathrm{~B}$ as the depth to the seafloor; for Hole 807A we subtracted 2815.1 $\mathrm{m}$; for Hole $807 \mathrm{C}$ we subtracted $2817.0 \mathrm{~m}$; and for Hole $586 \mathrm{C}$ we subtracted $2218.0 \mathrm{~m}$. We used Hole $806 \mathrm{~B}$ as our reference hole because shipboard biostratigraphy had already shown that this drill site had the highest sedimentation rate and so its record has the highest stratigraphic resolution.

To get the initial stratigraphic ties for the CORPAC inverse signal correlation package, we normally used a few of the biostratigraphic datum levels as initial depth ties between cores. For Holes 807A and $807 \mathrm{C}$, the biostratigraphic data was not needed as the holes are so close to each other and their sedimentation rates are almost the same. For the correlation of Hole $586 \mathrm{C}$ to Hole $806 \mathrm{~B}$, we used three nannofossil datum levels as initial tie-lines between the two cores: the last occurrence (LO) of Ceratolithus acutus (4.6 Ma), the LO of Discoaster quinqueramus (5.0 Ma), and the LO of Discoaster hamatus (8.7 Ma). We used two initial datum levels to tie Holes 807A and 806B: the LO of the foraminifer Globorotalia kugleri (20.9 Ma) and the LO of the nannofossil Discoaster quinqueramus (5.0 Ma).

\section{Sites 806 and 807}

The correlation between Holes $806 \mathrm{~B}$ and $807 \mathrm{~A}$ proved to be straightforward. Although we started by trying to correlate the SFLU shallow resistivity logs, we changed to correlating the medium penetration resistivity (IMPH) logs because the high resolution of the SFLU actually confused the correlation program. The sedimentation rate at Hole $807 \mathrm{~A}$ was sufficiently slower at Hole $806 \mathrm{~B}$ so that what appeared as one peak on the SFLU log at Hole 807A would be present as multiple peaks in Hole $806 \mathrm{~B}$. Nevertheless, we achieved a highquality correlation between the two records (Fig. 4). The record above $520 \mathrm{mbsf}$ (approximately $14.9 \mathrm{Ma}$ ) is easily matched between the two holes. Below this depth, however, the SFLU logs diverge from each other, as well as all other logs from the two drill sites. Figure 5 shows the velocity and SFLU logs for this interval. The velocity logs are offset from each other, because equivalent sedimentary ages are at different depths in the two cores, and sediment compaction adds a significant linear trend to the velocity data (Urmos et al., this volume). Except for the depth-related offset, small features above 520-550 mbsf can easily be correlated. Below this depth, the coherence between the two records is significantly smaller.

We were able to check our correlation by the independently determined biostratigraphic information (Fig. 6). The mapped correlation between Holes $807 \mathrm{~A}$ and $806 \mathrm{~B}$ connects the detailed nannofos- sil correlation scheme redone for this volume (Takayama and Backman, this volume). Thus, stratigraphy based upon the physical properties of the sediments at Sites 806 and 807 gives a correlation indistinguishable but in much more detail from the biostratigraphy in the two holes. The equivalent depths at Sites 807 and 806 can be found in Table 1.

\section{Sites 806 and 586}

The correlation between Holes 586C and 806B required little effort because the records almost perfectly match each other. We used the SFLU resistivity curves and were able to reach a coherence of 0.96 between the two logs with 19 harmonic coefficients (Fig. 7). Again the comparison to the biostratigraphy shows that this seems to be a reasonable correlation (Fig. 8). In this case, however, biostratigraphic information exists to only $300 \mathrm{mbsf}$ in the hole because the lower $300 \mathrm{~m}$ was washed and not cored. The deepest biostratigraphic point shown in Figure 7, that of the last occurrence of D. hamatus, is somewhat off the log correlation line, but the Leg 89 biostratigraphers noted that they only tentatively reached this datum (Moberly, Schlanger, et al., 1986). D. hamatus was extremely rare in the last core catcher and could have been reworked. The correlation listed in Table 1 would put the actual last occurrence of $D$. hamatus to be about $17 \mathrm{~m}$ deeper at Site 586. Here again, where a reasonable likelihood exists that the sediments at each drill site are similar, we have found that we can correlate the two sites in detail.

\section{Implications of the Correlation}

All the SFLU records from the sites on top of the Ontong Java Plateau contain a record of basically the same signal with only minor variations (Fig. 9), at least from approximately $14.9 \mathrm{Ma}$ to the top of our logging correlations, at $5 \mathrm{Ma}$. These sites are not adjacent to each other, but they span approximately $500 \mathrm{~m}$ in water depth and are as much as $485 \mathrm{~km}$ apart (Sites 807 and 806; see Fig. 1).

The relatively high frequency of the events implies that they should be driven by Milankovitch insolation cycles. Figure 10 shows a spectral analysis from a section between about 3.5 to $8.75 \mathrm{Ma}$ (based on biostratigraphy) of the shallow resistivity log from Hole 806B. This particular interval was assumed to have a constant sedimentation rate of $45.8 \mathrm{~m} / \mathrm{m}$.y. The record contains spectral power at or about the $120,95,41,23$, and 19 k.y. Milankovitch periods. Because the higher frequency 41 and 23 k.y. peaks are split, however, we think that the original time scale is overly simplified and that the sedimentation rate is not completely constant in this interval. By producing evolutionary spectra for this interval, we will be able to identify where the sedimentation rate changes occur and revise the time scale.

The Milankovitch orbital variations in solar insolation have in some manner affected the electrical resistivity of the sediment column. Because sediment resistivity is most strongly affected by porosity, we think that we are observing small-scale, coherent porosity variations from every drill site on top of the Ontong Java Plateau. We are uncertain what could have caused porosity to vary, but it may represent a grain-size effect. If so, the porosity variations could represent changes in winnowing caused by average current speeds over the plateau or by the average strength of tidal flow. We wish to reiterate, however, that the quality of the correlations across the entire top of the Ontong Java Plateau implies that the environmental changes that caused variations in porosity must be regional in scale. Local current variations could not have caused the observed patterns.

\section{Correlation of Plateau Flank Holes to Plateau Top Holes: Sites $803,805,806$, and 807}

Not surprisingly, the holes down the flank of the Ontong Java Plateau were more difficult to correlate to the reference drill site (Hole 806B) than holes from the top of the plateau. Because the 


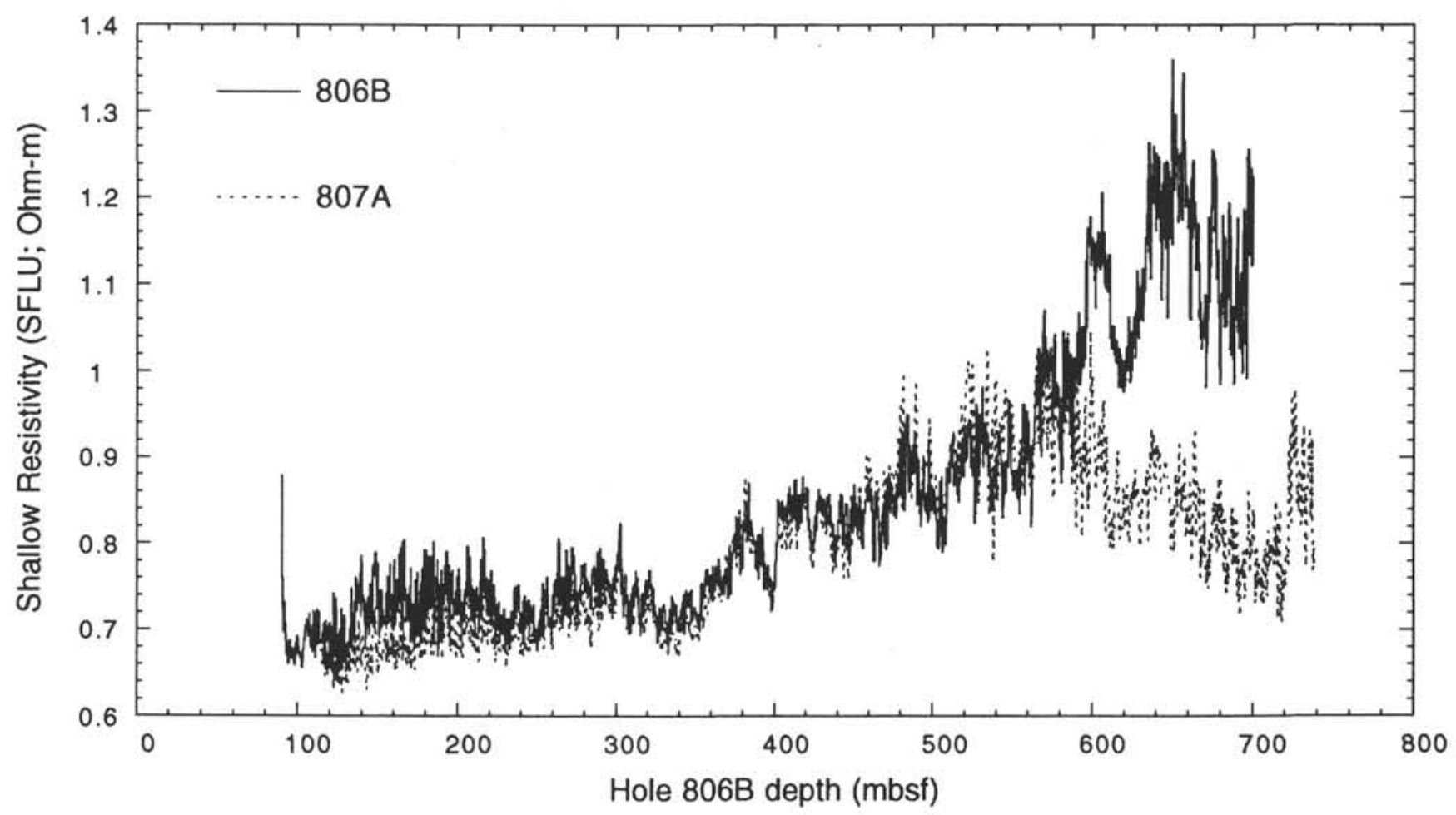

Figure 4. The SFLU shallow resistivity logs for Holes $806 \mathrm{~B}$ and $807 \mathrm{~A}$, about $485 \mathrm{~km}$ apart, plotted on the correlation depth scale of Hole $806 \mathrm{~B}$. Note how the upper part of the holes have a very high coherence, but the lower Miocene sections diverge, below $550 \mathrm{mbsf}$.

attempted correlation is of sediment physical properties, changes in sedimentation regime, whether through dissolution or through changes in deposition style, will strongly affect the records. Sites 805 $\left(1^{\circ} 13.7^{\prime} \mathrm{N}, 160^{\circ} 31.8^{\prime} \mathrm{E}, 3188 \mathrm{~m}\right.$ water depth) and $803\left(2^{\circ} 26.0^{\prime} \mathrm{N}\right.$, $160^{\circ} 32.5^{\prime} \mathrm{E}, 3412 \mathrm{~m}$ water depth) were cored as part of the depth transect down the flank of the plateau to examine water mass properties and to monitor fluctuations in carbonate dissolution with time. Site $804\left(1^{\circ} 00.3^{\prime} \mathrm{N}, 161^{\circ} 35.6^{\prime} \mathrm{E}, 3862 \mathrm{~m}\right.$ water depth) was not logged and will not be included in this report.

As explained below, we first attempted to correlate both sites to Site 806 by means of the resistivity records; however, we later decided that the bulk density logs contained more of a common signal. We also decided to correlate Site 803 to 807 , because Site 806 had insufficient record length for a good correlation, and because we found systematic differences in the physical properties of the northern cores (Sites 803 and 807) and the southern ones (Sites 586, 806, and $805)$. We will discuss these systematic differences in more detail later. The correlation of Sites 803 and 806 was then achieved by means of the Site $807 / 806$ correlation.

We found that correlations between Sites 806 and 805 based upon the SFLU resistivity suffered because of ambiguity in the logs from the deeper hole. The SFLU logs for the middle to late Miocene age section at Site 805 have about half the variance of Site 806, whereas the two bulk density logs are much more similar in amplitude. Thus, matching peaks is much easier when using bulk density logs. In the correlation of Sites 803 and 807 , we found low coherence between the SFLU logs. The best coherence we could obtain by correlating the SFLU logs was 0.50 (1.0 is perfect coherence-i.e., an exact match between records), whereas we achieved a coherence of 0.87 by correlating the bulk density logs and matched the independent biostratigraphic data better.

For Sites 806 and 803 , we used a combined data set of both discrete shipboard bulk density measurements and log data to make the bulk density profile. In both holes, the upper part of the holes had deteriorated before we were able to conduct the logging runs; they had washed out to be wider than the caliper holding the HLDT density tool against the borehole wall. The tool sensor then periodically rode away from the wall out into the borehole and logged a combination of borehole water and sediment. Low bulk density readings result from the washouts. Accordingly, we constructed composite sections by using shipboard physical properties to $235.8 \mathrm{mbsf}$ at Site 803 and to $320 \mathrm{mbsf}$ at Site 806 . Below these depths the logging bulk densities were used. The records were then interpolated to an even sample interval $(0.5-\mathrm{m}$ spacing at Site $806 ; 0.25-\mathrm{m}$ spacing at Site 803$)$ for the inverse signal correlation.

\section{Sites 806 and 805}

The results of the correlation are shown in Figures $11 \mathrm{~A}$ and 12 and in Table 1. The map between Sites 806 and 805 is not nearly so linear as those from the top of the plateau. Kinks in the correlation map are well constrained, however, as is shown in Figure 13. The match with biostratigraphy is very good, in part because we redid the correlation repeatedly when early versions did not match as well. The section most difficult to match was that at about 300 mbsf equivalent depth in Site 806 (Fig. 11A). The basic offset between the two records caused amplitudes of equivalent peaks to match poorly and made the correlation ambiguous. Nevertheless, with a sufficient number of trials, we did manage to achieve a believable correlation between the two drill sites.

\section{Sites 807 and 803}

Correlating Sites 807 and 803 by means of the bulk density profiles was straightforward (Fig. 11B). The results, shown in Figure 14 and in Table 2, match the calcareous nannofossil stratigraphy of Takayama and Backman (this volume). The section between 200 and $300 \mathrm{mbsf}$ in Hole $803 \mathrm{D}$ is offset from the biostratigraphic correlation between the two holes by about $10 \mathrm{~m}$, but we could not find any good way to change the bulk density correlation. 


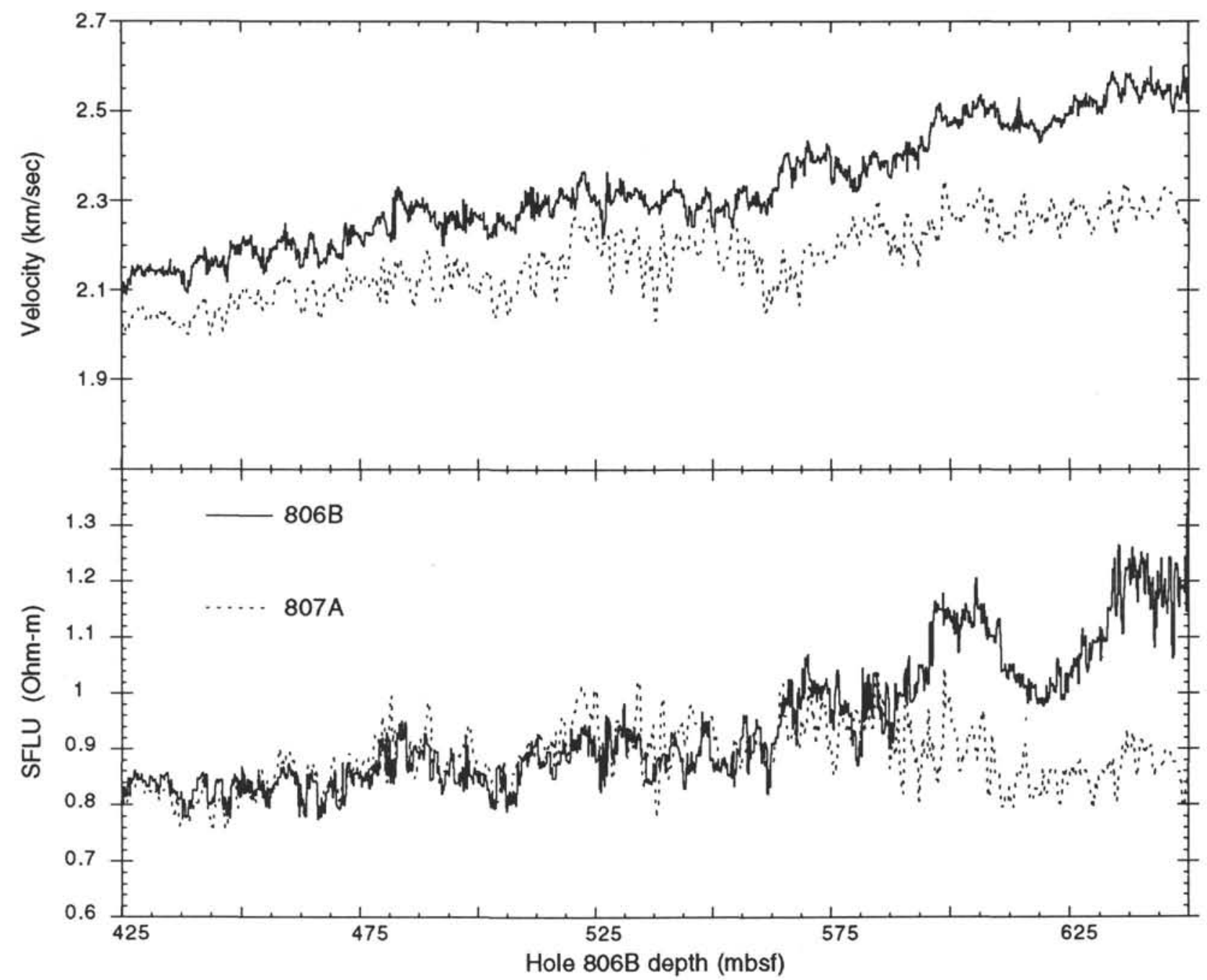

Figure 5 . A comparison between Holes $806 \mathrm{~B}$ and $807 \mathrm{~A}$ of the velocity and SFLU resistivity logs. The interval above about 520 mbsf is well correlated; below this depth (about $14.9 \mathrm{Ma}$ ), however, the records are divergent. It is unknown at this time why they diverge, but the cause may relate to the equatorial crossing of Site 807 at about $14.4 \mathrm{Ma}$.

\section{Latitudinal Effects upon Ontong Java Plateau Sedimentation}

We plotted two separate panels for Figure 11 to emphasize that, although these sites were drilled to be part of a single depth transect, obvious common latitudinal variations in sedimentation are present. Sites 807 and 803 are the two northernmost sites (Fig. 1), and they have a common bulk density profile marked most prominently by a flattening of the profile deeper than about $500 \mathrm{~m}$ equivalent depth in Site 806 . They also have higher bulk density excursions at $300 \mathrm{~m}$ and at about $420 \mathrm{~m}$ equivalent depth when compared with the southern sites. Sites to the south, including Site 586, have roughly linear bulk density profiles with depth and very low bulk density excursions. For this reason, when we compare fluctuations in sedimentation down the flank of the plateau in the next section, we compare Site 803 to Site 807 and the other drill sites to Site 806 .

\section{SEDIMENTATION RATE FLUCTUATIONS AND CCD CHANGES IN THE WESTERN EQUATORIAL PACIFIC OCEAN}

Part of the rationale for drilling the Leg 130 sites was to examine calcium carbonate dissolution through time for this western equatorial Pacific transect. The drill sites extend down the flank of the
Ontong Java Plateau from $2.6 \mathrm{~km}$ (the Holocene top of the lysocline) to just above the typical glacial top of the lysocline at about $3.6 \mathrm{~km}$ (Wu et al., 1991). Because the sediments are roughly $90 \%$ calcium carbonate, and because we can see little or no systematic variation of carbonate content down the flank of the plateau (Kroenke, Berger, Janecek, et al., 1991), relative sedimentation rates should to a first approximation reflect changes in calcite dissolution down the flanks. A relative sedimentation rate is the amount of sediment accumulated at one of the flank sites ( 805 and 803 ) divided by the amount of sediment accumulated during the same time period at a reference site on top of the plateau, where we assume that no dissolution has occurred. When the relative sediment accumulation equals 1 , the flank site and the reference site have the same sedimentation rate, and no dissolution has occurred, whereas a relative accumulation of 0 indicates that the flank site experienced a hiatus.

Figure 15 summarizes the variations we found. Plotted on the figure are the relative rates of accumulation at Sites 803 and 805 and CCD variations from the central Pacific for both equatorial DSDP drill sites (within $3^{\circ}$ of the equator) and nonequatorial sites. The CCD depth has been inverted on the figure, so that changes in the plotted CCD should have the same sense of change as changes in dissolution recorded by the relative sedimentation rates. Site 803 has changes in relative sediment accumulation that vaguely resemble changes in 


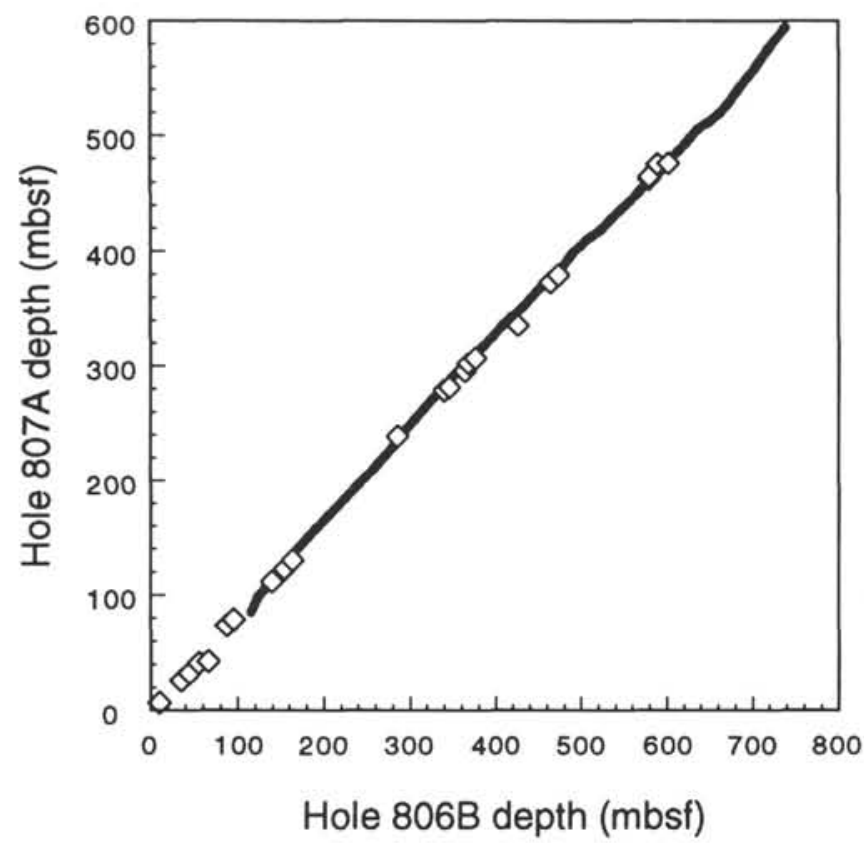

Figure 6. Correlation between Holes $806 \mathrm{~B}$ and $807 \mathrm{~A}$, showing the inverse signal correlation map (solid line) compared to the biostratigraphic events found from the detailed post-cruise analysis of nannofossils (Takayama and Backman, this volume).

equatorial CCD, at least for the last 40 m.y. of Earth's history. At about $34 \mathrm{Ma}$, when the Pacific experienced its deepest CCD, Site 803 accumulated almost as much sediment as Site 807 on top of the Ontong Java Plateau. The relative accumulation rate dropped through time so that by the early Miocene, at about $20 \mathrm{Ma}$, Site 803 was accumulating only about one third as much sediment as Site 807 . This was also a period when the $C C D$ was relatively deep. From the middle to late Miocene, the relative accumulation of sediment at Site 803 slowly increased. In this interval, however, the equatorial CCD has stayed relatively flat. If these changes of relative sediment accumulation represent changes in dissolution, the western equatorial Pacific has a different carbonate dissolution history than the central Pacific.

The relative accumulation history of Site 805 makes us question whether the sedimentation rates on the flank of the Ontong Java Plateau are controlled by carbonate dissolution. Because it is compared with Site 806 , we have only a Neogene record of relative sediment accumulation. Nevertheless, Site 805 has its own unique pattern of deposition. Roughly every 5 m.y., Site 805 went through a sedimentation cycle, alternating between low sedimentation rates, similar to Site 803 , and high sedimentation rates comparable to Site 806 , on top of the plateau. These cycles are insensitive to the correlation between Sites 806 and 805 . Repeated attempts to redo the correlation could not eliminate the cycles, and only managed to move their location in time slightly. At Site 805 two peaks of relatively high sediment accumulation occurred during the early Miocene interval during which Site 803 was accumulating sediments most slowly and when Site 804 experienced a hiatus. Thus, sediment accumulation patterns opposite in sign occurred within a $200-\mathrm{m}$ depth window. If this pattern is caused by dissolution, the lysocline must have become an extremely narrow horizon during these Miocene intervals.

The variations we have observed in relative sediment accumulation at Site 805 were not driven by cessation of sedimentation on top of the Ontong Java Plateau. Figure 16 plots sedimentation rates for Sites 803,805 , and 806 and the CCD profiles. The interval at about $20 \mathrm{Ma}$ at Site 806 is marked by the lowest sedimentation rates in the entire cored section, but the sedimentation rate never dropped below $10 \mathrm{~m} / \mathrm{m}$.y. The sedimentation rates at Site 805 oscillated fairly wildly during this time period. Thus, the oscillations at Site 805, not the sedimentation changes on top of the plateau, cause the pattern of relative sediment accumulation at Site 805 .

Comparing the complete profiles of sedimentation rate makes it clear that the absolute sedimentation rate over the entire Ontong Java Plateau was not driven by CCD changes but by other external factors in the carbon cycle. During the Miocene, when the central Pacific CCD experienced trends toward shallower CCDs (more dissolution), the sediments on the plateau first experienced a minimum in sedimentation centered about $20 \mathrm{Ma}$, and then experienced oscillatory, but generally increasing sedimentation rates throughout the rest of the period. Rates of inorganic carbon burial on the plateau increased dramatically, therefore, because the calcite contents of the sediments hardly varied from $90 \%$ (Kroenke, Berger, Janecek, et al., 1991).

\section{CONCLUSIONS}

Well logs have provided a means to correlate drill sites rapidly on the Ontong Java Plateau at a scale of meters or less. The logs are sufficiently reproducible to allow even subtle features to be correlated. On top of the plateau variations in sediment resistivity, which represent minor porosity variations, could be correlated across almost $500 \mathrm{~km}$ between sites. The quality of the correlations emphasize the uniform nature of sedimentation on top of the plateau and point out that winnowing events, if they occur, must be plateau-wide and not local sedimentation events.

We naively assumed, when we started this study, that correlating among drill sites down the flank of the Ontong Java Plateau would provide us with information that could easily be interpreted as dissolution variations caused by fluctuations in the western Pacific lysocline and CCD. We found, however, that some of the variations we observed were opposite in sign for adjacent sites. In particular, Site 803 , at $3.4 \mathrm{~km}$, and Site 805 , at $3.2 \mathrm{~km}$, accumulate sediment in different patterns through time. If the difference in sedimentation between these two sites is controlled largely by dissolution, then periods between 15 and $20 \mathrm{Ma}$ are present in which the lysocline must have thinned substantially, when there would have been almost a steplike transition between sediments that accumulated carbonate and those that did not. These "narrow lysocline" periods occur when Neogene sedimentation rates were the lowest; thus, they may represent a rising of the CCD caused by low inorganic carbon supply to the oceans. Alternatively, the change in relative sedimentation rates may be unrelated to deposition and may merely represent local variations in sediment deposition on the topographically complex flank of the Ontong Java Plateau. High-resolution interpretations of seismic reflection records along the flank of the plateau, or further drilling, will allow us to choose between these two alternatives.

\section{REFERENCES}

Berger, W.H., 1973. Cenozoic sedimentation in the eastern tropical Pacific. Geol. Soc. Am. Bull., 84:1941-1954.

Imbrie, J., Hays, J.D., Martinson, D.G., McIntyre, A., Mix, A.C., Morley, J.J., Pisias, N.G., Prell, W.L., and Shackleton, N.J., 1984. The orbital theory of Pleistocene climate: support from a revised chronology of the marine $\delta^{18} \mathrm{O}$ record. In Berger, A.L., Imbrie, J., Hays, J., Kukla, G., and Saltzman, B. (Eds.), Milankovitch and Climate (Pt. 1): Dordrecht (D. Reidel), 269-305.

Kroenke, L.W., Berger, W.H., Janecek, T.R., et al., 1991. Proc. ODP, Init. Repts., 130: College Station TX (Ocean Drilling Program).

Martinson, D.G., Menke, W., and Stoffa, P., 1982. An inverse approach to signal correlation. J. Geophys. Res., 87:4807-4818.

Martinson, D.G., Pisias, N.G., Hays, J.D., Imbrie, J., Moore, T.C., Jr., and Shackleton, N.J., 1987. Age dating and the orbital theory of the ice ages: development of a high resolution 0 to 300,000 -year chronostratigraphy. Quat. Res., 27:1-29.

Mayer, L.A., Shipley, T.H., Winterer, E.L., Mosher, D., and Hagen, R.A, 1991. SeaBeam and seismic reflection surveys on the Ontong Java Plateau. In Kroenke, L.W., Berger, W.H., Janecek, T.R., et al., 1991. Proc. ODP, Init. Repts., 130: College Station TX (Ocean Drilling Program), 45-75. 
Moberly, R., Schlanger, S.O., et al., 1986. Init. Repts. DSDP, 89: Washington (U.S. Govt. Printing Office).

Resig, J., Buyannanonth, V., and Roy, K., 1976. Foraminiferal stratigraphy and depositional history of the Ontong Java Plateau. Deep-Sea Res., 23:441-456.

Shackleton, N.J., et al., 1984. Oxygen isotope calibration of the onset of ice-rafting and history of glaciation in the North Atlantic region. Nature, 307:620-623.

van Andel, T.H., Heath, G.R., and Moore, T.C., Jr., 1975, Cenozoic history and paleoceanography of the central equatorial Pacific Ocean. Geol Soc. Am. Mem., 143. van Andel, T.H., and Moore, T.C., Jr., 1974. Cenozoic calcium carbonate distribution and calcite compensation depth in the central equatorial Pacific Ocean. Geology, 2:87-92.

Wu, G., Yasuda, M.K., and Berger, W.H., 1991. Late Pleistocene carbonate stratigraphy on Ontong-Java Plateau in the western equatorial Pacific. Mar. Geol., 99:135-150.

Date of initial receipt: 21 October 1991

Date of acceptance: 19 June 1992

Ms 130B-043

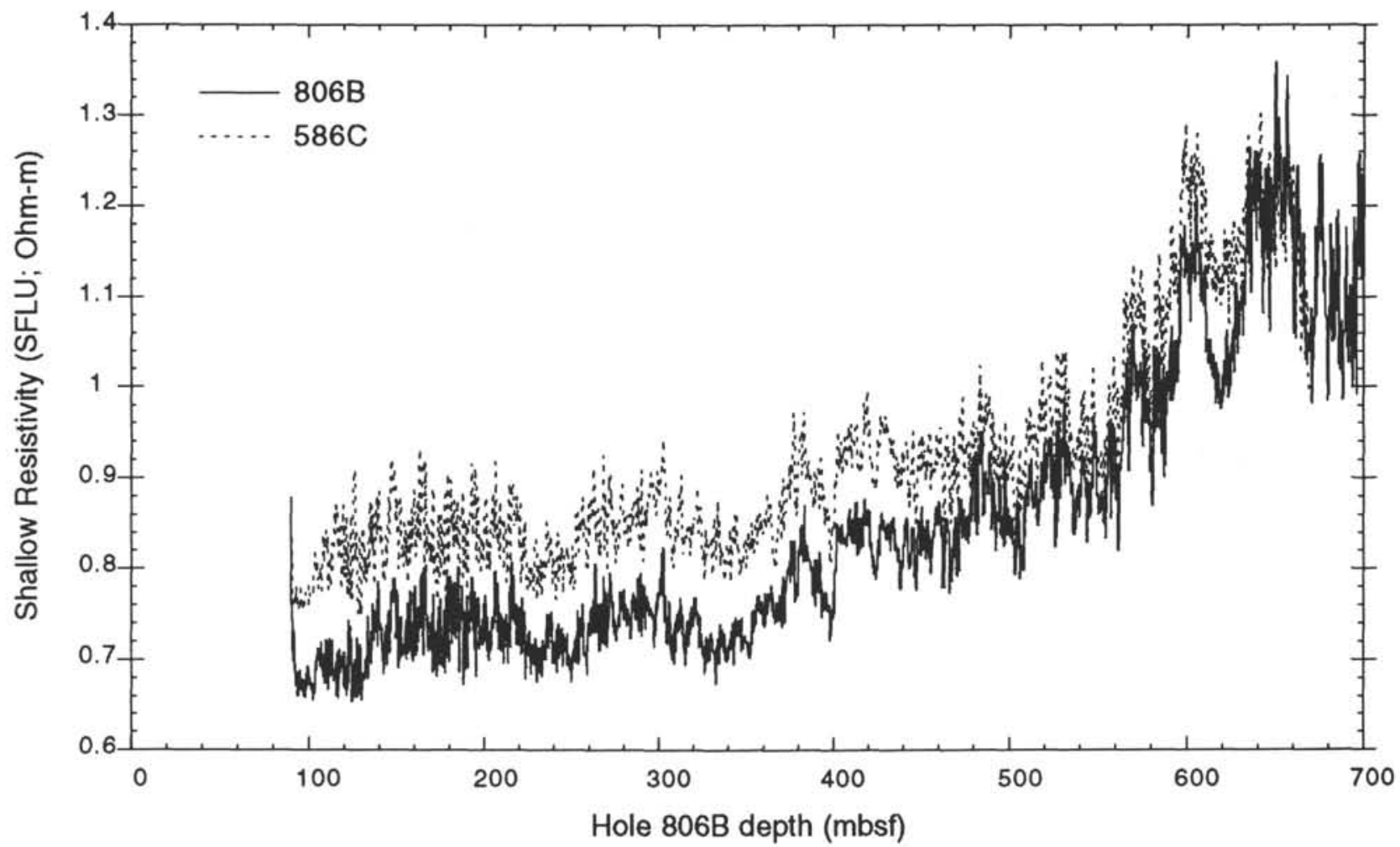

Figure 7. SFLU shallow resistivity well logs for Holes 806B and 586C, about $75 \mathrm{nmi}$ apart, plotted on the correlation depth scale of Hole $806 \mathrm{~B}$. Note the high coherence for the entire length of the records. The offset between the two logs is a result of uncorrected borehole size effects. 
Table 1. Equivalent depths at drill sites on the Ontong Java Plateau.

\begin{tabular}{|c|c|c|c|c|c|c|c|c|c|c|c|}
\hline \multicolumn{2}{|c|}{ Hole $806 \mathrm{~B}$} & \multirow{2}{*}{$\frac{\text { Hole } 586 \mathrm{C}}{\begin{array}{c}\text { Depth } \\
\text { (mbsf) }\end{array}}$} & \multirow{2}{*}{$\frac{\text { Hole 807A }}{\begin{array}{c}\text { Depth } \\
\text { (mbsf) }\end{array}}$} & \multirow{2}{*}{$\frac{\text { Hole } 805 \mathrm{C}}{\begin{array}{c}\text { Depth } \\
(\mathrm{mbsf})\end{array}}$} & \multirow{2}{*}{$\frac{\text { Hole 803D }}{\begin{array}{c}\text { Depth } \\
\text { (mbsf) }\end{array}}$} & Hole & 306 B & Hole $586 \mathrm{C}$ & Hole 807A & Hole $805 \mathrm{C}$ & Hole 803D \\
\hline $\begin{array}{l}\text { Depth } \\
\text { (mbsf) }\end{array}$ & $\begin{array}{l}\text { Age } \\
\text { (Ma) }\end{array}$ & & & & & $\begin{array}{l}\text { Depth } \\
\text { (mbsf) }\end{array}$ & $\begin{array}{l}\text { Age } \\
\text { (Ma) }\end{array}$ & $\begin{array}{l}\text { Depth } \\
\text { (mbsf) }\end{array}$ & $\begin{array}{l}\text { Depth } \\
\text { (mbsf) }\end{array}$ & $\begin{array}{l}\text { Depth } \\
\text { (mbsf) }\end{array}$ & $\begin{array}{l}\text { Depth } \\
\text { (mbsf) }\end{array}$ \\
\hline 90.0 & 3.426 & 95.55 & & & & 164.0 & 5.040 & 159.82 & 134.86 & 124.41 & \\
\hline 91.0 & 3.448 & 96.28 & & & & 165.0 & 5.062 & 160.66 & 136.08 & 125.19 & \\
\hline 92.0 & 3.469 & 96.85 & & & & 166.0 & 5.084 & 161.49 & 137.31 & 125.97 & \\
\hline 93.0 & 3.491 & 97.42 & & & & 167.0 & 5.105 & 162.39 & 138.32 & 126.74 & \\
\hline 94.0 & 3.513 & 97.99 & & & & 168.0 & 5.127 & 163.30 & 139.09 & 127.41 & 73.86 \\
\hline 95.0 & 3.535 & 98.55 & & & & 169.0 & 5.149 & 164.21 & 139.88 & 128.09 & 74.40 \\
\hline 96.0 & 3.557 & 99.12 & & & & 170.0 & 5.171 & 165.10 & 140.66 & 128.77 & 74.94 \\
\hline 97.0 & 3.578 & 99.69 & & & & 171.0 & 5.193 & 166.01 & 141.44 & 129.46 & 75.47 \\
\hline 98.0 & 3.600 & 100.26 & & & & 172.0 & 5.214 & 166.91 & 142.21 & 130.15 & 76.01 \\
\hline 99.0 & 3.622 & 100.84 & & & & 173.0 & 5.236 & 167.80 & 143.03 & 130.84 & 76.57 \\
\hline 100.0 & 3.644 & 101.41 & & & & 174.0 & 5.258 & 168.71 & 143.91 & 131.54 & 77.18 \\
\hline 101.0 & 3.666 & 101.98 & & & & 175.0 & 5.280 & 169.60 & 144.77 & 132.24 & 77.78 \\
\hline 102.0 & 3.688 & 102.55 & & & & 176.0 & 5.302 & 170.50 & 145.64 & 132.95 & 78.38 \\
\hline 103.0 & 3.709 & 103.12 & & & & 177.0 & 5.324 & 171.39 & 146.51 & 133.69 & 78.98 \\
\hline 104.0 & 3.731 & 103.69 & & & & 178.0 & 5.345 & 172.29 & 147.37 & 134.53 & 79.57 \\
\hline 105.0 & 3.753 & 104.56 & & & & 179.0 & 5.367 & 173.18 & 148,24 & 135.37 & 80.17 \\
\hline 106.0 & 3.775 & 105.53 & & & & 180.0 & 5.389 & 174.06 & 149.10 & 136.23 & 80.77 \\
\hline 107.0 & 3.797 & 106.51 & & & & 181.0 & 5.411 & 174.96 & 149.95 & 137.09 & 81.36 \\
\hline 108.0 & 3.818 & 107.48 & & & & 182.0 & 5.433 & 175.85 & 150.82 & 137.95 & 81.95 \\
\hline 109.0 & 3.840 & 108.46 & & & & 183.0 & 5.454 & 176.73 & 151.68 & 138.82 & 82.55 \\
\hline 110.0 & 3.862 & 109.43 & & & & 184.0 & 5.476 & 177.62 & 152.55 & 139.70 & 83.15 \\
\hline 111.0 & 3.884 & 110.41 & & & & 185.0 & 5.498 & 178.50 & 153.42 & 140.57 & 83.75 \\
\hline 112.0 & 3.906 & 111.40 & & & & 186.0 & 5.520 & 179.39 & 154.22 & 141.45 & 84.32 \\
\hline 113.0 & 3.927 & 112.37 & & & & 187.0 & 5.542 & 180.27 & 154.96 & 142.33 & 84.81 \\
\hline 114.0 & 3.949 & 113.36 & & & & 188.0 & 5.563 & 181.14 & 155.69 & 143.21 & 85.31 \\
\hline 115.0 & 3.971 & 114.34 & 84.92 & & & 189.0 & 5.585 & 182.04 & 156.43 & 144.08 & 85.83 \\
\hline 116.0 & 3.993 & 115.33 & 86.19 & & & 190.0 & 5.607 & 182.91 & 157.17 & 144.96 & 86.34 \\
\hline 117.0 & 4.015 & 116.31 & 87.46 & & & 191.0 & 5.629 & 183.79 & 157.91 & 145.76 & 86.85 \\
\hline 118.0 & 4.037 & 117.30 & 88.72 & & & 192.0 & 5.651 & 184.67 & 158.65 & 146.54 & 87.38 \\
\hline 119.0 & 4.058 & 118.29 & 90.22 & & & 193.0 & 5.673 & 185.54 & 159.44 & 147.31 & 87.92 \\
\hline 120.0 & 4.080 & 119.28 & 92.12 & & & 194.0 & 5.694 & 186.43 & 160.25 & 148.08 & 88.47 \\
\hline 121.0 & 4.102 & 120.28 & 94.01 & & & 195.0 & 5.716 & 187.31 & 161.05 & 148.84 & 89.03 \\
\hline 122.0 & 4.124 & 121.27 & 95.89 & & & 196.0 & 5.738 & 188.18 & 161.85 & 149.59 & 89.59 \\
\hline 123.0 & 4.146 & 122.26 & 97.76 & & & 197.0 & 5.760 & 189.06 & 162.66 & 150.33 & 90.16 \\
\hline 124.0 & 4.167 & 123.26 & 98.81 & & & 198.0 & 5.782 & 189.93 & 163.47 & 151.07 & 90.72 \\
\hline 125.0 & 4.189 & 124.26 & 99.69 & & & 199.0 & 5.803 & 190.81 & 164.29 & 151.82 & 91.27 \\
\hline 126.0 & 4.211 & 125.25 & 100.57 & & & 200.0 & 5.825 & 191.69 & 165.10 & 152.57 & 91.85 \\
\hline 1270 & 4.233 & 126.26 & 101.44 & & & 2010 & 5.847 & 192.56 & 165.92 & 153.30 & 92.42 \\
\hline 128.0 & 4.255 & 127.26 & 102.29 & & & 202.0 & 5.869 & 193.44 & 166.74 & 154.03 & 92.98 \\
\hline 129.0 & 4.276 & 128.27 & 103.14 & & & 203.0 & 5.891 & 194.32 & 167.54 & 154.74 & 93.56 \\
\hline 130.0 & 4.298 & 129.28 & 103.96 & & & 204.0 & 5.912 & 195.20 & 168.35 & 155.45 & 94.12 \\
\hline 131.0 & 4.320 & 130.28 & 104.78 & & & 205.0 & 5.934 & 196.07 & 169.15 & 156.14 & 94.67 \\
\hline 132.0 & 4.342 & 131.30 & 105.59 & & & 206.0 & 5.956 & 196.94 & 169.94 & 156.83 & 95.23 \\
\hline 133.0 & 4.364 & 132.30 & 106.39 & & & 207.0 & 5.978 & 197.83 & 170.73 & 157.51 & 95.78 \\
\hline 134.0 & 4.386 & 133.32 & 107.19 & & & 208.0 & 6.000 & 198.70 & 171.51 & 158.18 & 96.32 \\
\hline 135.0 & 4.407 & 134.34 & 107.97 & & & 209.0 & 6.022 & 199.58 & 172.30 & 158.85 & 96.87 \\
\hline 136.0 & 4.429 & 135.35 & 108.75 & & & 210.0 & 6.043 & 200.46 & 173.08 & 159.50 & 97.42 \\
\hline 137.0 & 4.451 & 136.38 & 109.54 & & & 211.0 & 6.065 & 201.32 & 173.86 & 160.15 & 97.97 \\
\hline 138.0 & 4.473 & 137.39 & 110.31 & & & 212.0 & 6.087 & 202.21 & 174.63 & 160.80 & 98.52 \\
\hline 139.0 & 4.495 & 138.41 & 111.07 & & & 213.0 & 6.109 & 203.08 & 175.46 & 161.44 & 99.08 \\
\hline 140.0 & 4.516 & 139.44 & 111.87 & & & 214.0 & 6.131 & 203.96 & 176.27 & 162.08 & 99.65 \\
\hline 141.0 & 4.538 & 140.30 & 112.59 & & & 215.0 & 6.152 & 204.84 & 177.06 & 162.72 & 100.21 \\
\hline 142.0 & 4.560 & 141.14 & 113.31 & & & 216.0 & 6.174 & 205.71 & 177.86 & 163.35 & 100.77 \\
\hline 143.0 & 4.582 & 141.99 & 114.03 & & & 217.0 & 6.196 & 206.55 & 178.66 & 163.98 & 101.34 \\
\hline 144.0 & 4.604 & 142.84 & 114.76 & & & 218.0 & 6.218 & 207.40 & 179.46 & 164.61 & 101.92 \\
\hline 145.0 & 4.625 & 143.68 & 115.49 & & & 219.0 & 6.240 & 208.24 & 180.25 & 165.24 & 102.48 \\
\hline 146.0 & 4.647 & 144.53 & 116.23 & & & 220.0 & 6.261 & 209.09 & 181.04 & 165.87 & 103.05 \\
\hline 147,0 & 4.669 & 145.38 & 117.35 & & & 221.0 & 6.283 & 209.94 & 181.83 & 166.50 & 103.62 \\
\hline 148.0 & 4.691 & 146.23 & 118.49 & & & 222.0 & 6.305 & 210.78 & 182.62 & 167.13 & 104.19 \\
\hline 149.0 & 4.713 & 147.08 & 119.66 & & & 223.0 & 6.327 & 211.62 & 183.40 & 167.77 & 104.75 \\
\hline 150.0 & 4.735 & 147.93 & 120.89 & & & 224.0 & 6.349 & 212.48 & 184.19 & 168.41 & 105.30 \\
\hline 151.0 & 4.756 & 148.78 & 122.12 & & & 225.0 & 6.371 & 213.32 & 184.97 & 169.05 & 105.87 \\
\hline 152.0 & 4.778 & 149.63 & 123.29 & & & 226.0 & 6.392 & 214.16 & 185.75 & 169.70 & 106.43 \\
\hline 153.0 & 4.800 & 150.47 & 124.08 & & & 227.0 & 6.414 & 215.02 & 186.52 & 170.35 & 106.98 \\
\hline 154.0 & 4.822 & 151.33 & 124.88 & & & 228.0 & 6.436 & 215.86 & 187.29 & 171.01 & 107.53 \\
\hline 155.0 & 4.844 & 152.18 & 125.69 & & & 229.0 & 6.458 & 216.71 & 188.07 & 171.68 & 108.08 \\
\hline 156.0 & 4.865 & 153.03 & $|26.5|$ & & & 230.0 & 6.480 & 217.56 & 188.84 & 172.35 & 108.64 \\
\hline 157.0 & 4.887 & 153.87 & 127.34 & & & 231.0 & 6.501 & 218.40 & 189.60 & 173.03 & 109.19 \\
\hline 158.0 & 4.909 & 154.73 & 128.18 & & & 232.0 & 6.523 & 219.24 & 190.37 & 173.71 & 109.74 \\
\hline 159.0 & 4.931 & 155.58 & 129.03 & & & 233.0 & 6.545 & 220.10 & 191.13 & 174.41 & 110.29 \\
\hline 160.0 & 4.953 & 156.43 & 130.02 & & & 234.0 & 6.567 & 220.94 & 191.89 & 175.11 & 110.84 \\
\hline 161.0 & 4.975 & 157.27 & 131.21 & & & 235.0 & 6.589 & 221.79 & 192.65 & 175.82 & 111.37 \\
\hline 162.0 & 4.996 & 158.12 & 132.43 & 122.86 & & 236.0 & 6.610 & 222.64 & 193,42 & 176.54 & 111.92 \\
\hline 163.0 & 5.018 & 158.96 & 133.64 & 123.64 & & 237.0 & 6.632 & 223.48 & 194.16 & 177.27 & 112.46 \\
\hline
\end{tabular}


Table 1 (continued).

\begin{tabular}{|c|c|c|c|c|c|c|c|c|c|c|c|}
\hline \multicolumn{2}{|c|}{ Hole 806B } & \multirow{2}{*}{ 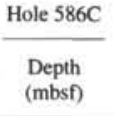 } & \multirow{2}{*}{ 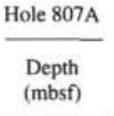 } & \multirow{2}{*}{$\frac{\text { Hole } 805 \mathrm{C}}{\mathcal{C} \begin{array}{c}\text { Depth } \\
(\mathrm{mbsf})\end{array}}$} & \multirow{2}{*}{$\frac{\text { Hole 803D }}{\begin{array}{c}\text { Depth } \\
\text { (mbsf) }\end{array}}$} & Hole & 06B & Hole 586C & Hole 807A & Hole 805C & Hole 803D \\
\hline $\begin{array}{l}\text { Depth } \\
\text { (mbsf) }\end{array}$ & $\begin{array}{l}\text { Age } \\
\text { (Ma) }\end{array}$ & & & & & $\begin{array}{l}\text { Depth } \\
\text { (mbsf) }\end{array}$ & $\begin{array}{l}\text { Age } \\
\text { (Ma) }\end{array}$ & $\begin{array}{l}\text { Depth } \\
\text { (mbsf) }\end{array}$ & $\begin{array}{l}\text { Depth } \\
\text { (mbsf) }\end{array}$ & $\begin{array}{l}\text { Depth } \\
\text { (mbsf) }\end{array}$ & $\begin{array}{l}\text { Depth } \\
\text { (mbsf) }\end{array}$ \\
\hline 238.0 & 6.654 & 224.33 & 194.92 & 178.01 & 113.00 & 312.0 & 8.268 & 288.01 & 259.00 & 234.18 & 161.71 \\
\hline 239.0 & 6.676 & 225.18 & 195.72 & 178.76 & 113.56 & 313.0 & 8.290 & 288.90 & 259.89 & 235.07 & 162.26 \\
\hline 240.0 & 6.698 & 226.02 & 196.53 & 179.51 & 114.14 & 314.0 & 8.312 & 289.80 & 260.81 & 235.96 & 162.84 \\
\hline 241.0 & 6.720 & 226.86 & 197.32 & 180.28 & 114.71 & 315.0 & 8.334 & 290.69 & 261.70 & 236.84 & 163.41 \\
\hline 242.0 & 6.741 & 227.72 & 198.11 & 181.05 & 115.27 & 316.0 & 8.356 & 291.58 & 262.60 & 237.72 & 163.98 \\
\hline 243.0 & 6.763 & 228.56 & 198.89 & 181.83 & 115.83 & 317.0 & 8.377 & 292.48 & 263.48 & 238.59 & 164.55 \\
\hline 244.0 & 6.785 & 229.41 & 199.70 & 182.62 & 116.39 & 318.0 & 8.399 & 293.36 & 264.35 & 239.45 & 165.11 \\
\hline 245.0 & 6.807 & 230.25 & 200.48 & 183.42 & 116.95 & 319.0 & 8.421 & 294.26 & 265.23 & 240.38 & 165.68 \\
\hline 246.0 & 6.829 & 231.09 & 201.26 & 184.21 & 117.51 & 320.0 & 8.443 & 295.14 & 266.09 & 241.44 & 166.24 \\
\hline 247.0 & 6.850 & 231.94 & 202.04 & 184.99 & 118.07 & 321.0 & 8.465 & 296.03 & 266.97 & 242.49 & 166.81 \\
\hline 248.0 & 6.872 & 232.79 & 202.83 & 185.78 & 118.62 & 322.0 & 8.486 & 296.92 & 267.83 & 243.53 & 167.36 \\
\hline 249.0 & 6.894 & 233.63 & 203.61 & 186.57 & 119.17 & 323.0 & 8.508 & 297.79 & 268.72 & 244.56 & 167.95 \\
\hline 250.0 & 6.916 & 234.48 & 204.38 & 187.37 & 119.72 & 324.0 & 8.530 & 298.68 & 269.53 & 245.59 & 168.48 \\
\hline 251.0 & 6.938 & 235.33 & 205.16 & 188.17 & 120.28 & 325.0 & 8.552 & 299.57 & 270.29 & 246.58 & 168.96 \\
\hline 252.0 & 6.959 & 236.17 & 205.95 & 188.97 & 120.82 & 326.0 & 8.574 & 300.45 & 271.05 & 247.46 & 169.48 \\
\hline 253.0 & 6.981 & 237.01 & 206.72 & 189.78 & 121.36 & 327.0 & 8.595 & 301.34 & 271.83 & 248.31 & 169.99 \\
\hline 254.0 & 7.003 & 237.86 & 207.39 & 190.59 & 121.84 & 328.0 & 8.617 & 302.21 & 272.62 & 249.15 & 170.51 \\
\hline 255.0 & 7.025 & 238.71 & 208.03 & 191.40 & 122.28 & 329.0 & 8.639 & 303,09 & 273.42 & 249.90 & 171.04 \\
\hline 256.0 & 7.047 & 239.55 & 208.68 & 192.21 & 122.75 & 330.0 & 8.661 & 303.98 & 274.23 & 250.61 & 171.59 \\
\hline 257.0 & 7.069 & 240.40 & 209.51 & 193.03 & 123.32 & 331.0 & 8.683 & 304.85 & 275.06 & 251.60 & 172.14 \\
\hline 258.0 & 7.090 & 241.25 & 210.38 & 193.84 & 123.94 & 332.0 & 8.705 & 305.73 & 275.91 & 252.59 & 172.70 \\
\hline 259.0 & 7.112 & 242.09 & 211.24 & 194.65 & 124.53 & 333.0 & 8.726 & 306.62 & 276.75 & 253.58 & 173.27 \\
\hline 260.0 & 7.134 & 242.95 & 212.09 & 195.47 & 125.14 & 334.0 & 8.760 & 307.49 & 277.60 & 254.57 & 173.84 \\
\hline 261.0 & 7.156 & 243.79 & 212.95 & 196.28 & 125.72 & 335.0 & 8.802 & 308.38 & 278.47 & 255.57 & 174.42 \\
\hline 262.0 & 7.178 & 244.64 & 213.79 & 197.09 & 126.31 & 336,0 & 8.844 & 309.25 & 279.34 & 256.57 & 175.01 \\
\hline 263.0 & 7.199 & 245.50 & 214.63 & 197.90 & 126.90 & 337.0 & 8.885 & 310.13 & 280.22 & 257.58 & 175.61 \\
\hline 264.0 & 7.221 & 246.34 & 215.48 & 198.71 & 127.49 & 338.0 & 8.927 & 311,01 & 281.10 & 258.59 & 176.21 \\
\hline 265.0 & 7.243 & 247.20 & 216.32 & 199.52 & 128.07 & 339.0 & 8.969 & 311.88 & 281.98 & 260.19 & 176.81 \\
\hline 266.0 & 7.265 & 248.05 & 217.17 & 200.33 & 128.66 & 340.0 & 9.011 & 312.77 & 282.86 & 262.07 & 177.41 \\
\hline 267.0 & 7.287 & 248.90 & 218.01 & 201.14 & 129.24 & 341.0 & 9.052 & 313.64 & 283.75 & 263.96 & 178.01 \\
\hline 268.0 & 7.309 & 249.74 & 218.87 & 201.94 & 129.83 & 342.0 & 9.094 & 314.53 & 284.72 & 265.43 & 178.68 \\
\hline 269.0 & 7.330 & 250.60 & 219.71 & 202.75 & 130.56 & 343.0 & 9.136 & 315.42 & 285.69 & 266.40 & 179.34 \\
\hline 270.0 & 7.352 & 251.46 & 220.58 & 203.55 & 131.33 & 344.0 & 9.178 & 316.29 & 286.65 & 267.28 & 179.99 \\
\hline 271.0 & 7.374 & 252.32 & 221.43 & 204.40 & 132.10 & 345.0 & 9.220 & 317.17 & 287.48 & 267.80 & 180.58 \\
\hline 272.0 & 7.396 & 253.17 & 222.30 & 205.26 & 132.88 & 346.0 & 9.261 & 318.06 & 288.29 & 268.33 & 181.12 \\
\hline 273.0 & 7,418 & 254.02 & 223.20 & 206.12 & 133.67 & 347.0 & 9.303 & 318.93 & 289.10 & 268.87 & 181.68 \\
\hline 274.0 & 7.439 & 254.88 & 224.10 & 206.98 & 134.49 & 348.0 & 9.345 & 319.83 & 289.89 & 269.40 & 182.22 \\
\hline 275.0 & 7.461 & 255.74 & 225.02 & 207.84 & 135.32 & 349.0 & 9.387 & 320.71 & 290.68 & 269.93 & 182.76 \\
\hline 276.0 & 7.483 & 256.59 & 225.95 & 208.69 & 136.14 & 350.0 & 9.429 & 321.59 & 291.45 & 270.47 & 183.30 \\
\hline 277.0 & 7.505 & 257.45 & 226.88 & 209.55 & 136.98 & 351.0 & 9.470 & 322.48 & 292.23 & 271.00 & 183,84 \\
\hline 278.0 & 7.527 & 258.32 & 227.83 & 210.31 & 137.81 & 352.0 & 9.512 & 323.36 & 293.00 & 271.54 & 184.38 \\
\hline 279.0 & 7.548 & 259.17 & 228.79 & 210.75 & 138.67 & 353.0 & 9.554 & 324.25 & 293.76 & 272.07 & 184.89 \\
\hline 280.0 & 7.570 & 260.04 & 229.72 & 211.20 & 139.51 & 354.0 & 9.596 & 325.14 & 294.51 & 272.60 & 185.42 \\
\hline 281.0 & 7.592 & 260.90 & 230.66 & 211.64 & 140.33 & 355.0 & 9.637 & 326.02 & 295.26 & 273.12 & 185.94 \\
\hline 282.0 & 7.614 & 261.76 & 231.61 & 212.08 & 141.20 & 356.0 & 9.679 & 326.92 & 296.01 & 273.65 & 186.46 \\
\hline 283.0 & $\begin{array}{l}7.636 \\
7.650\end{array}$ & 262.62 & 232.59 & 212.52 & 142.05 & 357.0 & 9.721 & 327.81 & 296.76 & 274.17 & 186.97 \\
\hline 284.0 & 7.658 & 263.48 & 233.57 & 212.97 & 142.92 & 358.0 & 9.763 & 328.70 & 297.51 & 274.65 & 187.49 \\
\hline 285.0 & 7.679 & 264.35 & 234.57 & 213.41 & 143.82 & 359.0 & 9,805 & 329.59 & 298.27 & 275.08 & 188.01 \\
\hline 286.0 & 7.701 & 265.21 & 235.57 & 213.85 & 144.70 & 360.0 & 9.846 & 330.49 & 299.02 & 275.50 & 188.54 \\
\hline 287.0 & 7.723 & 266.07 & 236.52 & 214.29 & 145.54 & 361.0 & 9.888 & 331.39 & 299.78 & 275.93 & 189.08 \\
\hline 288.0 & 7.745 & 266.94 & 237.43 & 214.73 & 146.36 & 362.0 & 9.930 & 332.27 & 300.55 & 276.35 & 189.60 \\
\hline 289.0 & 7.767 & 267.80 & 238.37 & 215.30 & 147.21 & 363.0 & 9.972 & 333.17 & 301.33 & 276.77 & 190.14 \\
\hline 290.0 & 7.788 & 268.66 & 239.31 & 215.87 & 148.03 & 364.0 & 10.013 & 334.07 & 302.11 & 277.19 & 190.68 \\
\hline 291.0 & 7.810 & 269.52 & 240.25 & 216.44 & 148.87 & 365.0 & 10.055 & 334.97 & 302.90 & 277.60 & 191.22 \\
\hline 292.0 & 7.832 & 270.39 & 241.21 & 217.01 & 149.73 & 366.0 & 10.097 & 335.87 & 303.72 & 278.02 & 191.79 \\
\hline 293.0 & 7.854 & 271.25 & 242.17 & 217.57 & 150.59 & 367.0 & 10.139 & 336.76 & 304.53 & 278.44 & 192.35 \\
\hline 294.0 & 7.876 & 272.11 & 243.12 & 218.14 & 151.44 & 368.0 & 10.181 & 337.67 & 305.29 & 278.85 & 192.87 \\
\hline 295.0 & 7.897 & 272.97 & 244.08 & 218.70 & 152.29 & 369.0 & 10.222 & 338.56 & 305.98 & 279.26 & 193.35 \\
\hline 296.0 & 7.919 & 273.83 & 245.03 & 219.26 & 153.09 & 370.0 & 10.264 & 339.47 & 306.66 & 279.68 & 193.84 \\
\hline 297.0 & 7.941 & 274.70 & 245.98 & 219.82 & 153.65 & 371.0 & 10.306 & 340.37 & 307.36 & 280.09 & 194.31 \\
\hline 298.0 & 7.963 & 275.55 & 246.91 & 220.38 & 154,21 & 372.0 & 10.348 & 341.27 & 308.07 & 280.50 & 194.78 \\
\hline 299.0 & 7.985 & 276.41 & 247.81 & 221.01 & 154.76 & 373.0 & 10.390 & 342.18 & 308.78 & 280.92 & 195.28 \\
\hline 300.0 & 8.007 & 277.27 & 248.65 & 221.65 & 155.28 & 374.0 & 10.431 & 343.08 & 309.49 & 281.33 & 195.74 \\
\hline 301.0 & 8.028 & 278.12 & 249.47 & 222.80 & 155.76 & 375.0 & 10.473 & 343.99 & 310.21 & 281.75 & 196.14 \\
\hline 302.0 & 8.050 & 278.99 & 250.32 & 223.94 & 156.30 & 376.0 & 10.515 & 344.89 & 310.92 & 282.17 & 196.48 \\
\hline 303.0 & 8.072 & 279.89 & 251.29 & 225.08 & 156.90 & 377.0 & 10.557 & 345.81 & 311.63 & 282.59 & 196.86 \\
\hline 304.0 & 8.094 & 280.79 & 252.22 & 226.21 & 157.46 & 378.0 & 10.598 & 346.71 & 312.35 & 283.01 & 197.24 \\
\hline 305.0 & 8.116 & 281.70 & 253.14 & 227.34 & 158.03 & 379.0 & 10.640 & 347.62 & 313.06 & 283.44 & 197.60 \\
\hline 306.0 & 8.137 & 282.60 & 254.02 & 228.46 & 158.58 & 380.0 & 10.682 & 348.53 & 313.78 & 283.87 & 197.98 \\
\hline 307.0 & 8.159 & 283.51 & 254.90 & 229.57 & 159.13 & 381.0 & 10.724 & 349.44 & 314.49 & 284.30 & 198.34 \\
\hline 308.0 & 8.181 & 284.41 & 255.75 & 230.54 & 159.66 & 382.0 & 10.766 & 350.35 & 315.20 & 284.73 & 198.71 \\
\hline 309.0 & 8.203 & 285.31 & 256.58 & 231.46 & 160.19 & 383.0 & 10.807 & 351.26 & 315.90 & 285.17 & 199.09 \\
\hline 310.0 & 8.225 & 286.22 & 257.41 & 232.37 & 160.70 & 384.0 & 10.849 & 352.18 & 316.61 & 285.62 & 199.46 \\
\hline 311.0 & 8.246 & 287.11 & 258.22 & 233.28 & 161.20 & 385.0 & 10.891 & 353.08 & 317.31 & 286.06 & 199.82 \\
\hline
\end{tabular}


Table 1 (continued).

\begin{tabular}{|c|c|c|c|c|c|c|c|c|c|c|c|}
\hline \multicolumn{2}{|c|}{ Hole 806B } & \multirow{2}{*}{$\frac{\text { Hole 586C }}{\begin{array}{c}\text { Depth } \\
\text { (mbsf) }\end{array}}$} & \multirow{2}{*}{$\frac{\text { Hole 807A }}{\begin{array}{c}\text { Depth } \\
\text { (mbsf) }\end{array}}$} & \multirow{2}{*}{$\frac{\text { Hole } 805 \mathrm{C}}{\begin{array}{c}\text { Depth } \\
\text { (mbsf) }\end{array}}$} & \multirow{2}{*}{$\frac{\text { Hole 803D }}{\begin{array}{c}\text { Depth } \\
\text { (mbsf) }\end{array}}$} & Hole & 806B & Hole $586 \mathrm{C}$ & Hole $807 \mathrm{~A}$ & Hole $805 \mathrm{C}$ & Hole 803D \\
\hline $\begin{array}{l}\text { Depth } \\
\text { (mbsf) }\end{array}$ & $\begin{array}{l}\text { Age } \\
\text { (Ma) }\end{array}$ & & & & & $\begin{array}{l}\text { Depth } \\
\text { (mbsf) }\end{array}$ & $\begin{array}{l}\text { Age } \\
(\mathrm{Ma})\end{array}$ & $\begin{array}{l}\text { Depth } \\
\text { (mbsf) }\end{array}$ & $\begin{array}{l}\text { Depth } \\
\text { (mbsf) }\end{array}$ & $\begin{array}{l}\text { Depth } \\
\text { (mbsf) }\end{array}$ & $\begin{array}{l}\text { Depth } \\
\text { (mbsf) }\end{array}$ \\
\hline 386.0 & 10.933 & 354.00 & 318.01 & 286.51 & 200.19 & 513.0 & 14.524 & 469.70 & 413.24 & 360.15 & 242.01 \\
\hline 387.0 & 10.975 & 354.91 & 318.71 & 286.97 & 200.55 & 514.0 & 14.582 & 470.61 & 413.76 & 361.20 & 242.18 \\
\hline 388.0 & 11.016 & 355.82 & 319.40 & 287.43 & 200.91 & 515.0 & 14.640 & 471.54 & 414.27 & 362.39 & 242.37 \\
\hline 389.0 & 11.058 & 356.73 & 320.10 & 287.89 & 201.27 & 516.0 & 14.697 & 472.45 & 414.77 & 363.58 & 242.56 \\
\hline 390.0 & 11.100 & 357.64 & 320.80 & 288.36 & 201.64 & 517.0 & 14.755 & 473.37 & 415.28 & 364.77 & 242.74 \\
\hline 391.0 & 11.142 & 358.56 & 321.50 & 288.83 & 202.00 & 518.0 & 14.813 & 474.27 & 415.79 & 365.94 & 242.91 \\
\hline 392.0 & 11.183 & 359.47 & 322.20 & 289.31 & 202.36 & 519.0 & 14.870 & 475.19 & 416.31 & 367.10 & 243.10 \\
\hline 393.0 & 11.225 & 360.39 & 322.89 & 289.79 & 202.73 & 520.0 & 14.928 & 476.11 & 416.81 & 368.24 & 243.28 \\
\hline 394.0 & 11.267 & 361.29 & 323.60 & 290.27 & 203.09 & 521.0 & 14.986 & 477.03 & 417.32 & 369.34 & 243.46 \\
\hline 395.0 & 11.309 & 362.21 & 324.30 & 290.76 & 203.45 & 522.0 & 15.043 & 477.95 & 417.87 & 370.04 & 243.67 \\
\hline 396.0 & 11.351 & 363.12 & 325.01 & 291.25 & 203.81 & 523.0 & 15.101 & 478.86 & 418.66 & 370.52 & 243.95 \\
\hline 397.0 & 11.392 & 364.03 & 325.71 & 291.74 & 204.19 & 524.0 & 15.159 & 479.79 & 419.46 & 370.99 & 244.25 \\
\hline 398.0 & 11.434 & 364.94 & 326.42 & 292.23 & 204.55 & 525.0 & 15.217 & 480.70 & 420.27 & 371.44 & 244.54 \\
\hline 399.0 & 11.476 & 365.85 & 327.24 & 292.73 & 204.95 & 526.0 & 15.274 & 481.63 & 421.07 & 371.88 & 244.83 \\
\hline 400.0 & 11.518 & 366.77 & 328.14 & 293.23 & 205.43 & 527.0 & 15.332 & 482.55 & 421.88 & 372.30 & 245.13 \\
\hline 401.0 & 11.559 & 367.67 & 329.02 & 293.72 & 205.88 & 528.0 & 15.390 & 483.47 & 422.68 & 372.70 & 245.41 \\
\hline 402.0 & 11.601 & 368.59 & 329.89 & 294.22 & 206.33 & 529.0 & 15.447 & 484.39 & 423.48 & 373.09 & 245.71 \\
\hline 403.0 & 11.643 & 369.50 & 330.77 & 294.72 & 206.77 & 530.0 & 15.505 & 485.31 & 424.29 & 373.45 & 246.00 \\
\hline 404.0 & 11.685 & 370.40 & 331.63 & 295.22 & 207.21 & 531.0 & 15.563 & 486.24 & 425.08 & 373.85 & 246.30 \\
\hline 405.0 & 11.727 & 371.33 & 332.51 & 295.71 & 207.66 & 532.0 & 15.620 & 487.17 & 425.88 & 374.23 & 246.60 \\
\hline 406.0 & 11.768 & 372.23 & 333.37 & 296.21 & 208.10 & 533.0 & 15.678 & 488.10 & 426.68 & 374.60 & 246.89 \\
\hline 407.0 & 11.810 & 373.14 & 334.24 & 296.71 & 208.54 & 534.0 & 15.736 & 489.02 & 427.48 & 374.95 & 247.18 \\
\hline 408.0 & 11.852 & 374.05 & 335.10 & 297.20 & 208.98 & 535.0 & 15.793 & 489.96 & 428.27 & 375.28 & 247.48 \\
\hline 461.0 & 13.350 & 422.14 & 374.09 & 325.48 & 227.82 & 536.0 & 15.851 & 490.88 & 429.06 & 375.59 & 247.78 \\
\hline 462.0 & 13.366 & 423.04 & 374.73 & 326.04 & 228.05 & 537.0 & 15.909 & 491.82 & 429.80 & 375.89 & 248.05 \\
\hline 463.0 & 13.383 & 423.97 & 375.35 & 326.59 & 228.29 & 538.0 & 15.966 & 492.74 & 430.52 & 376.18 & 248.33 \\
\hline 464.0 & 13.399 & 424.88 & 375.97 & 327.14 & 228.53 & 539.0 & 16.004 & 493.67 & 431.25 & 376.45 & 248.60 \\
\hline 465.0 & 13.415 & 425.80 & 376.62 & 327.68 & 228.77 & 540.0 & 16.043 & 494.60 & 431.97 & 376.72 & 248.88 \\
\hline 466.0 & 13.431 & 426.71 & 377.31 & 329.16 & 229.03 & 541.0 & 16.081 & 495.54 & 432.69 & 376.97 & 249.14 \\
\hline 467.0 & 13.447 & 427.63 & 378.01 & 330.85 & 229.29 & 542.0 & 16.119 & 496.47 & 433.41 & 377.21 & 249.41 \\
\hline 468.0 & 13.464 & 428.54 & 378.72 & 332.49 & 229.56 & 543.0 & 16.158 & 497.39 & 434.12 & 377.45 & 249.69 \\
\hline 469.0 & 13.480 & 429,46 & 379.44 & 334.07 & 229.83 & 544.0 & 16.196 & 498.33 & 434.83 & 377.67 & 249.96 \\
\hline 470.0 & 13.496 & 430.38 & 380.16 & 335.61 & 230.10 & 545.0 & 16.234 & 499.26 & 435.54 & 377.89 & 250.23 \\
\hline 471.0 & 13.512 & 431.29 & 380.91 & 337.11 & 230.38 & 546.0 & 16.272 & 500.20 & 436.25 & 378.11 & 250.51 \\
\hline 472.0 & 13.528 & 432.22 & 381.69 & 338.56 & 230.67 & 547.0 & 16.311 & 501.13 & 436.96 & 378.31 & 250.78 \\
\hline 473.0 & 13.545 & 433.12 & 382.48 & 339.84 & 230.97 & 548.0 & 16.349 & 502.06 & 437.66 & 378.52 & 251.05 \\
\hline 474.0 & 13.561 & 434.05 & 383.28 & 340.00 & 231.26 & 549.0 & 16.387 & 502.98 & 438.37 & 378.71 & 251.32 \\
\hline 475.0 & 13.577 & 434.96 & 384.10 & 340.26 & 231.57 & 550.0 & 16.426 & 503.92 & 439.07 & 378.91 & 251.59 \\
\hline 476.0 & 13.593 & 435.88 & 384.91 & 340.50 & 231.86 & 551.0 & 16.464 & 504.85 & 439.77 & 379.10 & 251.86 \\
\hline 477.0 & 13.609 & 436.80 & 385.76 & 340.74 & 232.17 & 552.0 & 16.502 & 505.78 & 440.46 & 379.29 & 252.13 \\
\hline 478.0 & 13.626 & 437.71 & 386.60 & 340.98 & 232.49 & 553.0 & 16.540 & 506.71 & 441.15 & 379.48 & 252.40 \\
\hline 479.0 & 13.642 & 438.64 & 387.45 & 341.22 & 232.80 & 554.0 & 16.579 & 507.63 & 441.85 & 379.66 & 252.67 \\
\hline 480.0 & 13.658 & 439.55 & 388.44 & 341.46 & 233.14 & 555.0 & 16.617 & 508.57 & 442.54 & 379.85 & 252.93 \\
\hline 481.0 & 13.674 & 440.47 & 389.48 & 341.69 & 233.53 & 556.0 & 16.655 & 509.49 & 443.22 & 380.03 & 253.22 \\
\hline 482.0 & 13.690 & 441.39 & 390.50 & 341.98 & 233.90 & 557.0 & 16.694 & 510.41 & 443.91 & 380.21 & 253.55 \\
\hline 483.0 & 13.707 & 442.31 & 391.53 & 342.32 & 234.27 & 558.0 & 16.732 & 511.33 & 444.59 & 380.39 & 253.87 \\
\hline 484.0 & 13.723 & 443.22 & 392.52 & 342.67 & 234.63 & 559.0 & 16.770 & 512.26 & 445.30 & 380.63 & 254.20 \\
\hline 485.0 & 13.739 & 444.14 & 393.52 & 343.02 & 234.99 & 560.0 & 16.808 & 513.17 & 446.01 & 380,88 & 254.53 \\
\hline 486.0 & 13.755 & 445.05 & 394.49 & 343.37 & 235.34 & 561.0 & 16.847 & 514.10 & 446.72 & 381.13 & 254.87 \\
\hline 487.0 & 13.771 & 445.98 & 395.44 & 343.73 & 235.67 & 562.0 & 16.885 & 515.02 & 447.42 & 381.39 & 255.21 \\
\hline 488.0 & 13.788 & 446.89 & 396.36 & 344.11 & 236.00 & 563.0 & 16.923 & 515.93 & 448.12 & 381.65 & 255.54 \\
\hline 489.0 & 13.804 & 447.80 & 397.27 & 344.49 & 236.33 & 564,0 & 16.961 & 516.85 & 448.83 & 381.92 & 255.87 \\
\hline 490.0 & 13.820 & 448.72 & 398.14 & 344.89 & 236.64 & 565.0 & 17.000 & 517.77 & 449.55 & 382.23 & 256.18 \\
\hline 491.0 & 13.836 & 449.63 & 399.00 & 345.30 & 236.94 & 566.0 & 17.038 & 518.77 & 450.34 & 382.55 & 256.42 \\
\hline 492.0 & 13.852 & 450.54 & 399.76 & 345.73 & 237.21 & 567.0 & 17.076 & 519.78 & 451.13 & 382.88 & 256.62 \\
\hline 493.0 & 13.869 & 451.46 & 400.45 & 346.06 & 237.46 & 568.0 & 17.115 & 520.78 & 451.92 & 383.22 & 256.85 \\
\hline 494.0 & 13.885 & 452.37 & 401.11 & 346.27 & 237.69 & 569.0 & 17.153 & 521.78 & 452.70 & 383.57 & 257.07 \\
\hline 495.0 & 13.901 & 453.29 & 401.77 & 346.63 & 237.92 & 570.0 & 17.191 & 522.77 & 453.48 & 383.93 & 257.30 \\
\hline 496.0 & 13.917 & 454.20 & 402.41 & 347.23 & 238.15 & 571.0 & 17.229 & 523.77 & 454.27 & 384.31 & 257.51 \\
\hline 497.0 & 13.933 & 455.11 & 403.04 & 347.85 & 238.37 & 572.0 & 17.268 & 524.76 & 454.91 & 384.70 & 257.69 \\
\hline 498.0 & 13.950 & 456.02 & 403.67 & 348,48 & 238.60 & 573.0 & 17.306 & 525.75 & 455.43 & 385.10 & 257.85 \\
\hline 499.0 & 13.966 & 456.94 & 404.27 & 349.14 & 238.84 & 574.0 & 17.344 & 526.74 & 455.95 & 385.51 & 258.00 \\
\hline 500.0 & 13.982 & 457.85 & 405.08 & 349.80 & 239.10 & 575.0 & 17.383 & 527.73 & 456.48 & 385.93 & 258.14 \\
\hline 501.0 & 13.998 & 458.77 & 405.87 & 350.49 & 239.39 & 576.0 & 17.421 & 528.72 & 457.00 & 386.36 & 258.29 \\
\hline 502.0 & 14.014 & 459.68 & 406.65 & 351.20 & 239.66 & 577.0 & 17.459 & 529.70 & 457.52 & 386.79 & 258.44 \\
\hline 503.0 & 14.031 & 460.59 & 407.42 & 351.93 & 239.93 & 578.0 & 17.497 & 530.69 & 458.04 & 387.24 & 258.59 \\
\hline 504.0 & 14.047 & 461.50 & 408.17 & 352.67 & 240.20 & 579.0 & 17.536 & 531.66 & 458.56 & 387.68 & 258.74 \\
\hline 505.0 & 14.063 & 462.40 & 408.92 & 353.44 & 240.46 & 580.0 & 17.574 & 532.65 & 459.09 & 388.14 & 258.89 \\
\hline 506.0 & 14.121 & 463.32 & 409.66 & 354.22 & 240.72 & 581.0 & 17.612 & 533.62 & 459.61 & 388.60 & 259.03 \\
\hline 507.0 & 14.178 & 464.23 & 410.21 & 355.02 & 240,94 & 582.0 & 17.650 & 534.60 & 460.13 & 389.59 & 259.18 \\
\hline 508.0 & 14.236 & 465.15 & 410.72 & 355.84 & 241.11 & 583.0 & 17.689 & 535.58 & 460.65 & 390.59 & 259.32 \\
\hline 509.0 & 14.294 & 466.05 & 411.23 & 356.68 & 241.28 & 584.0 & 17.727 & 536.55 & 461.17 & 391.54 & 259.47 \\
\hline 510.0 & 14.351 & 466.97 & 411.73 & 357.53 & 241.46 & 585.0 & 17.765 & 537.52 & 461.76 & 392.11 & 259.66 \\
\hline 511.0 & 14.409 & 467.89 & 412.23 & 358.39 & 241.65 & 586.0 & 17.804 & 538.49 & 462.78 & 392.67 & 259.92 \\
\hline 512.0 & 14.467 & 468.79 & 412.74 & 359.27 & 241.83 & 587.0 & 17.842 & 539.47 & 463.80 & 393.23 & 260.21 \\
\hline
\end{tabular}


Table 1 (continued).

\begin{tabular}{|c|c|c|c|c|c|c|c|c|c|c|c|}
\hline \multicolumn{2}{|c|}{ Hole 806B } & \multirow{2}{*}{$\frac{\text { Hole } 586 \mathrm{C}}{\mathcal{T}_{\text {Depth }}}$} & \multirow{2}{*}{$\frac{\text { Hole 807A }}{\begin{array}{c}\text { Depth } \\
\text { (mbsf) }\end{array}}$} & \multirow{2}{*}{$\frac{\text { Hole 805C }}{\begin{array}{c}\text { Depth } \\
\text { (mbsf) }\end{array}}$} & Hole 803D & Hole & 306B & Hole $586 \mathrm{C}$ & Hole $807 \mathrm{~A}$ & Hole $805 \mathrm{C}$ & Hole 803D \\
\hline $\begin{array}{l}\text { Depth } \\
\text { (mbsf) }\end{array}$ & $\begin{array}{l}\text { Age } \\
\text { (Ma) }\end{array}$ & & & & $\begin{array}{c}\text { Depth } \\
\text { (mbsf) }\end{array}$ & $\begin{array}{l}\text { Depth } \\
\text { (mbsf) }\end{array}$ & $\begin{array}{l}\text { Age } \\
\text { (Ma) }\end{array}$ & $\begin{array}{l}\text { Depth } \\
\text { (mbsf) }\end{array}$ & $\begin{array}{l}\text { Depth } \\
\text { (mbsf) }\end{array}$ & $\begin{array}{l}\text { Depth } \\
\text { (mbsf) }\end{array}$ & $\begin{array}{l}\text { Depth } \\
\text { (mbsf) }\end{array}$ \\
\hline 588.0 & 17.880 & 540.44 & 464.82 & 393.78 & 260.50 & 664.0 & 22.568 & 616.39 & 520.84 & 449.67 & 279.79 \\
\hline 589.0 & 17.918 & 541.41 & 465.84 & 394.34 & 260.78 & 665.0 & 22.610 & 617.39 & 521.71 & 450.29 & 280.12 \\
\hline 590.0 & 17.957 & 542.38 & 466.86 & 394.89 & 261.07 & 666.0 & 22.652 & 618.40 & 522.57 & 450.90 & 280.47 \\
\hline 591.0 & 17.995 & 543.35 & 467.88 & 395.43 & 261.36 & 667.0 & 22.694 & 619.42 & 523.43 & 451.50 & 280.81 \\
\hline 592.0 & 18.033 & 544.32 & 468.91 & 395.97 & 261.64 & 668.0 & 22.736 & 620.42 & 524.29 & 452.10 & 281.15 \\
\hline 593.0 & 18.072 & 545.30 & 469.92 & 396.51 & 261.93 & 669.0 & 22.778 & 621.40 & 525.14 & 452.68 & 281.50 \\
\hline 594.0 & 18.138 & 546.26 & 470.94 & 397.05 & 262.20 & 670.0 & 22.820 & 622.37 & 526.00 & 453.26 & 281.85 \\
\hline 595.0 & 18.204 & 547.24 & 471.97 & 397.60 & 262.49 & 671.0 & 22.862 & & 526.85 & 453.84 & 282.19 \\
\hline 596.0 & 18.270 & 548.20 & 472.98 & 398.21 & 262.76 & 672.0 & 22.904 & & 527.79 & 454,41 & 282.58 \\
\hline 597.0 & 18.336 & 549.17 & 474.01 & 398.77 & 263.05 & 673.0 & 22.946 & & 528.92 & 454.97 & 283.04 \\
\hline 598.0 & 18.402 & 550.15 & 475.02 & 399.34 & 263.33 & 674.0 & 22.988 & & 530.05 & 455.54 & 283.50 \\
\hline 599.0 & 18.468 & 551.11 & 476.05 & 399.90 & 263.61 & 675.0 & 23.030 & & 531.17 & 456.13 & 283.96 \\
\hline 600.0 & 18.534 & 552.09 & 476.85 & 400.46 & 263.83 & 676.0 & 23.072 & & 532.29 & 456.73 & 284.46 \\
\hline 601.0 & 18.600 & 553.06 & 477.53 & 401.03 & 264.01 & 677.0 & 23.114 & & 533.30 & 457.33 & 285.19 \\
\hline 602.0 & 18.666 & 554.04 & 478.21 & 401.59 & 264.20 & 678.0 & 23.156 & & 534.31 & 457.92 & 285.87 \\
\hline 603.0 & 18.732 & 555.01 & 478.89 & 402.17 & 264.40 & 679.0 & 23.199 & & 535.32 & 458.52 & 286.56 \\
\hline 604.0 & 18.798 & 556.00 & 479.58 & 402.74 & 264.63 & 680.0 & 23.241 & & 536.34 & 459.12 & 287.26 \\
\hline 605.0 & 18.864 & 556.96 & 480.25 & 403.33 & 264.88 & 681.0 & 23.283 & & 537.36 & 459.72 & 287.96 \\
\hline 606.0 & 18.930 & 557.95 & 480.95 & 403.92 & 265.13 & 682.0 & 23.325 & & 538.37 & 460.35 & 288.66 \\
\hline 607.0 & 18.996 & 558.92 & 481.66 & 404.52 & 265.39 & 683.0 & 23.367 & & 539.39 & 461.00 & 289.36 \\
\hline 608.0 & 19.062 & 559.91 & 482.42 & 405.13 & 265.66 & 684.0 & 23.409 & & 540.40 & 461.66 & 290.07 \\
\hline 609.0 & 19.128 & 560.89 & 483.18 & 405.76 & 265.94 & 685.0 & 23.451 & & 541.42 & 462.32 & 290.77 \\
\hline 610.0 & 19.194 & 561.88 & 483.93 & 406.41 & 266.21 & 686.0 & 23.493 & & 542.44 & 462.98 & 291.48 \\
\hline 611.0 & 19.260 & 562.86 & 484.70 & 407.07 & 266.48 & 687.0 & 23.535 & & 543.41 & 463.65 & 292.15 \\
\hline 612.0 & 19.326 & 563.85 & 485.47 & 407.74 & 266.76 & 688.0 & 23.577 & & 544.37 & 464.33 & 292.82 \\
\hline 613.0 & 19.392 & 564.84 & 486.23 & 408.44 & 267.03 & 689.0 & 23.619 & & 545.32 & 465.01 & 293.48 \\
\hline 614.0 & 19.458 & 565.83 & 486.99 & 409.16 & 267.31 & 690.0 & 23.661 & & 546.27 & 465.70 & 294.14 \\
\hline 615.0 & 19.524 & 566.82 & 487.76 & 409.91 & 267.58 & 691.0 & 23.703 & & 547.23 & 466.43 & 294.82 \\
\hline 616.0 & 19.590 & 567.81 & 488.55 & 410.68 & 267.86 & 692.0 & 23.745 & & 548.20 & 467.28 & 295.48 \\
\hline 617.0 & 19.656 & 568.81 & 489.33 & 411.48 & 268.14 & 693.0 & 23.787 & & 549.16 & 468.14 & 296.15 \\
\hline 618.0 & 19.722 & 569.80 & 490.11 & 412.31 & 268.42 & 694.0 & 23.830 & & 550.13 & 469.00 & 296.82 \\
\hline 619.0 & 19.788 & 570.80 & 490.89 & 413.17 & 268.70 & 695.0 & 23.872 & & 551.09 & 469.87 & 297.50 \\
\hline 620.0 & 19.854 & 571.81 & 491.68 & 414.07 & 268.98 & 696.0 & 23.914 & & 552.07 & 470.76 & 298.18 \\
\hline 621.0 & 19.920 & 572.81 & 492.48 & 415.00 & 269.26 & 697.0 & 23.956 & & 553.04 & 471.63 & 298.85 \\
\hline 622.0 & 19.986 & 573.81 & 493.27 & 415.97 & 269.55 & 698.0 & 23.998 & & 553.89 & 472.50 & 299.44 \\
\hline 623.0 & 20.052 & 574.81 & 494.07 & 416.97 & 269.84 & 699.0 & 24.040 & & 554.74 & 473.39 & 300.00 \\
\hline 624.0 & 20.118 & 575.82 & 494.89 & 417.99 & 270.13 & 700.0 & 24.082 & & 555.58 & 474.29 & 300.63 \\
\hline 625.0 & 20.184 & 576.82 & 495.70 & 419.01 & 270.41 & 701.0 & 24.124 & & 556.68 & 475.19 & 301.35 \\
\hline 626.0 & 20.250 & 577.84 & 496.51 & 419.98 & 270.70 & 702.0 & 24.166 & & 557.79 & 476.10 & 302.10 \\
\hline 627.0 & 20.316 & 578.85 & 497.33 & 420.97 & 271.00 & 703.0 & 24.208 & & 558.89 & 477.02 & 302.86 \\
\hline 628.0 & 20.382 & 579.85 & 498.17 & 421.99 & 271.30 & 704.0 & 24.250 & & 560.00 & 477.93 & 303.61 \\
\hline 629.0 & 20.448 & 580.87 & 499.00 & 423.04 & 271.60 & 705.0 & 24.292 & & 561.12 & 478.86 & 304.37 \\
\hline 630.0 & 20.514 & 581.88 & 499.83 & 424.10 & 271.90 & 706.0 & 24.334 & & 562.23 & 479.78 & 305.11 \\
\hline 631.0 & 20.580 & 582.90 & 500.68 & 425.19 & 272.20 & 707.0 & 24.376 & & 563.33 & & 305.86 \\
\hline 632.0 & 20.647 & 583.92 & 501.52 & 426.29 & 272.51 & 708.0 & 24.418 & & 564.45 & & 306.60 \\
\hline 633.0 & 20.713 & 584.92 & 502.37 & 427,40 & 272.82 & 709.0 & 24.460 & & 565.56 & & 307.34 \\
\hline 634.0 & 20.779 & 585.95 & 503.23 & 428.52 & 273.14 & 709.0 & 24.460 & & 565.56 & & 307.34 \\
\hline 635.0 & 20.845 & 586.97 & 504.09 & 429.64 & 273.45 & 710.0 & 24.503 & & 566.67 & & 308.06 \\
\hline 636.0 & 20.911 & 587.98 & 504.96 & 430.77 & 273.77 & 711.0 & 24.545 & & 567.76 & & 308.80 \\
\hline 637.0 & 20.977 & 589.00 & 505.57 & 431.89 & 274,00 & 712.0 & 24.587 & & 568.88 & & 309.51 \\
\hline 638.0 & 21.043 & 590.01 & 506.09 & 432.99 & 274.18 & 713.0 & 24.629 & & 569.98 & & 310.24 \\
\hline 639.0 & 21.109 & 591.03 & 506.62 & 433.71 & 274.38 & 714.0 & 24.671 & & 571.08 & & 310.95 \\
\hline 640.0 & 21.175 & 592.05 & 507.15 & 434.38 & 274.57 & 715.0 & 24.713 & & 572.17 & & 311.65 \\
\hline 641.0 & 21.241 & 593.07 & 507.63 & 435.04 & 274.75 & 716.0 & 24.755 & & 573.27 & & 312.34 \\
\hline 642.0 & 21.307 & 594.09 & 508.10 & 435.69 & 274.92 & 717.0 & 24.797 & & 574.20 & & 312.96 \\
\hline 643.0 & 21.373 & 595.10 & 508.57 & 436.32 & 275.09 & 718.0 & 24.839 & & 575.07 & & 313.62 \\
\hline 644.0 & 21.439 & 596.12 & 509.05 & 436.94 & 275.27 & 719.0 & 24.881 & & 575.99 & & 314.21 \\
\hline 645.0 & 21.505 & 597.15 & 509.53 & 437.54 & 275.45 & 720.0 & 24.923 & & 576.96 & & 314.83 \\
\hline 646.0 & 21.571 & 598.16 & 509.99 & 438.12 & 275.63 & 721.0 & 24.965 & & 577.90 & & 315.44 \\
\hline 647.0 & 21.637 & 599.18 & 510.48 & 438.69 & 275.80 & 722.0 & 25.007 & & 578.86 & & 316.04 \\
\hline 648.0 & 21.703 & 600.19 & 510.95 & 439.24 & 275.98 & 723.0 & 25.049 & & 579.81 & & 316.64 \\
\hline 649.0 & 21.769 & 601.21 & 511.43 & 439.77 & 276.16 & 724.0 & 25.091 & & 580.76 & & 317.24 \\
\hline 650.0 & 21.835 & 602.23 & 511.90 & 440.29 & 276.34 & 725.0 & 25.134 & & 581.69 & & 317.82 \\
\hline 651.0 & 21.901 & 603.24 & 512.38 & 440.79 & 276.52 & 726.0 & 25.176 & & 582.65 & & 318.41 \\
\hline 652.0 & 21.967 & 604.26 & 512.94 & 441.28 & 276.73 & 727.0 & 25.218 & & 583.59 & & 319.00 \\
\hline 653.0 & 22.033 & 605.26 & 513.60 & 441.89 & 276.98 & 728.0 & 25.260 & & 584.54 & & 319.58 \\
\hline 654.0 & 22.099 & 606.28 & 514.26 & 442.69 & 277.24 & 729.0 & 25.302 & & 585.47 & & 320.17 \\
\hline 655.0 & 22.165 & 607.29 & 514.90 & 443.47 & 277.49 & 730.0 & 25.344 & & 586.42 & & 320.74 \\
\hline 656.0 & 22.231 & 608.31 & 515.56 & 444.23 & 277.73 & 731.0 & 25.386 & & 587.35 & & 321.32 \\
\hline 657.0 & 22.273 & 609.32 & 516.21 & 444.97 & 277.98 & 732.0 & 25.428 & & 588.29 & & 321.89 \\
\hline 658.0 & 22.315 & 610.33 & 516.86 & 445.69 & 278.24 & 733.0 & 25.470 & & 589.22 & & 322.45 \\
\hline 659.0 & 22.357 & 611.34 & 517.50 & 446.39 & 278.48 & 734.0 & 25.512 & & 590.15 & & 323.02 \\
\hline 660.0 & 22.399 & 612.35 & 518.15 & 447.08 & 278.73 & 735.0 & 25.554 & & 591.08 & & 323.58 \\
\hline 661.0 & 22.441 & 613.36 & 518.79 & 447.74 & 278.98 & 736.0 & 25.596 & & 592.00 & & 324.15 \\
\hline 662.0 & 22.483 & 614.36 & 519.44 & 448.40 & 279.23 & 737.0 & 25.638 & & 592.94 & & 324.72 \\
\hline 663.0 & 22.526 & 615.38 & 520.08 & 449.04 & 279.48 & 738.0 & 25.680 & & 593.96 & & 325.35 \\
\hline
\end{tabular}

Note: Depths are based upon the well-log correlation between Hole 806B and Holes 586C, 807A, 805C, and 803D. The preliminary age scale is for Hole 806B. Correlation to Hole $803 \mathrm{D}$ is based upon the correlation between Holes 806B and 807A and between Holes 803D and 807A. 


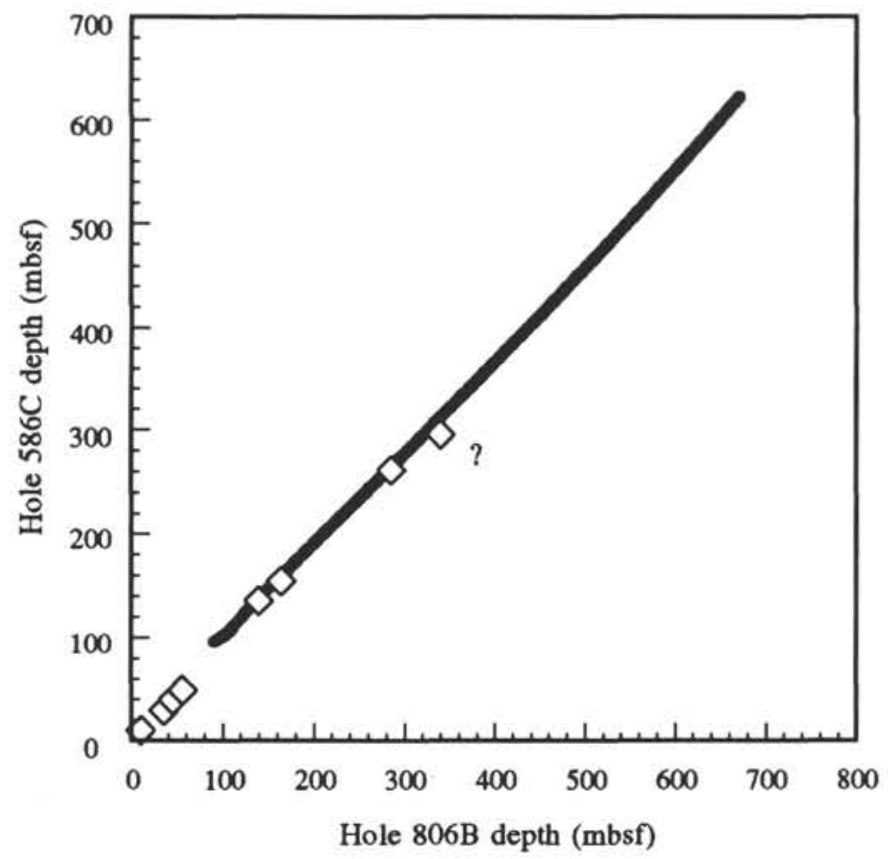

Figure 8 . Stratigraphic correlation between Holes $806 \mathrm{~B}$ and $586 \mathrm{C}$, showing the inverse signal correlation map (solid line) compared to the nannofossil biostratigraphic events identified from the detailed post-cruise analysis (Takayama and Backman, this volume).

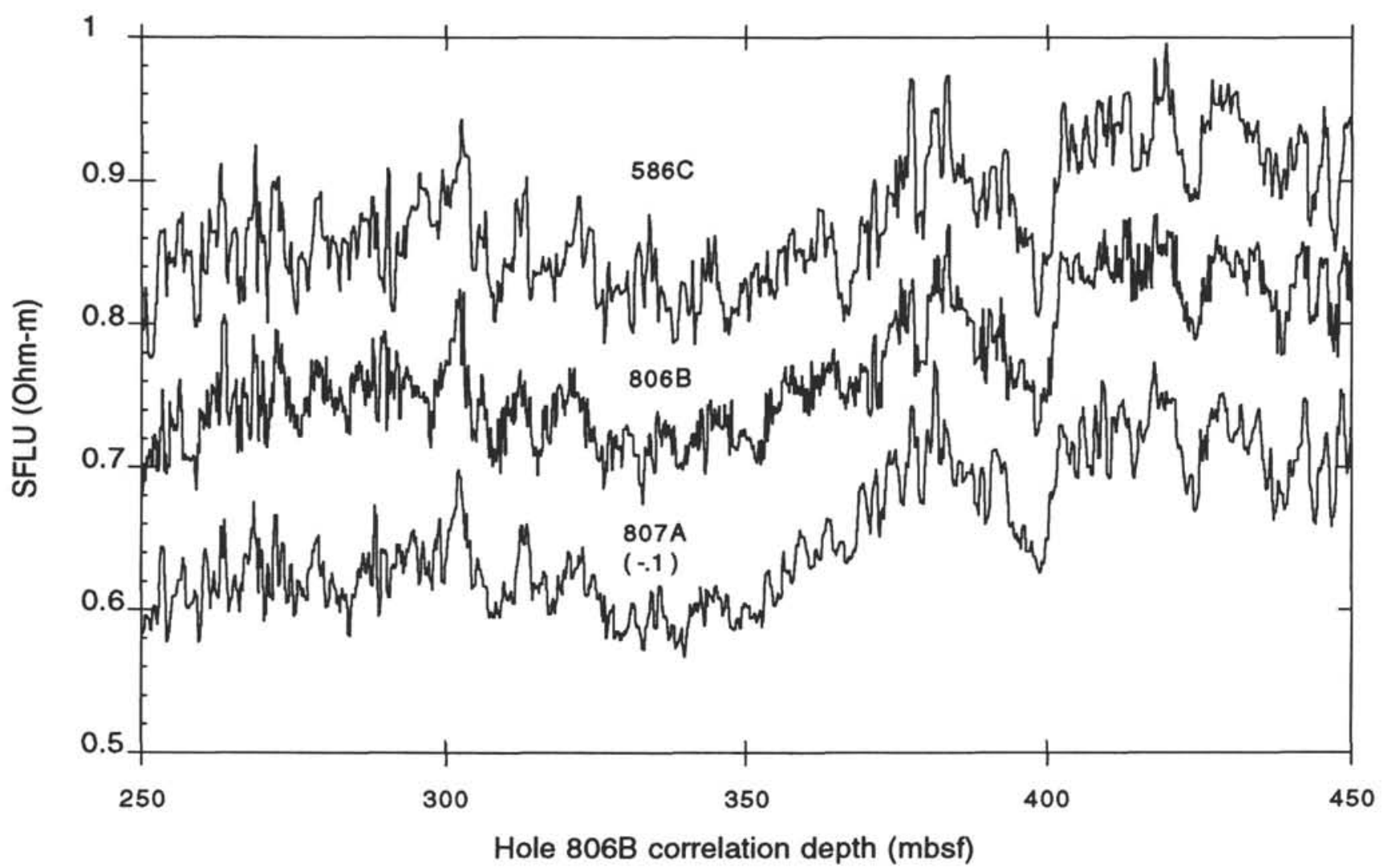

Figure 9. A section of the resistivity data from all the holes on top of the Ontong Java Plateau depth-shifted to the equivalent depth in Hole 806B. The records have been offset with respect to each other for clarity. Resistivity variations are probably a result of small-scale porosity changes between sedimentary beds that must have great lateral extent to be correlated between Holes $807 \mathrm{~A}$ and $806 \mathrm{~B}$, almost $500 \mathrm{~km}$ apart. 


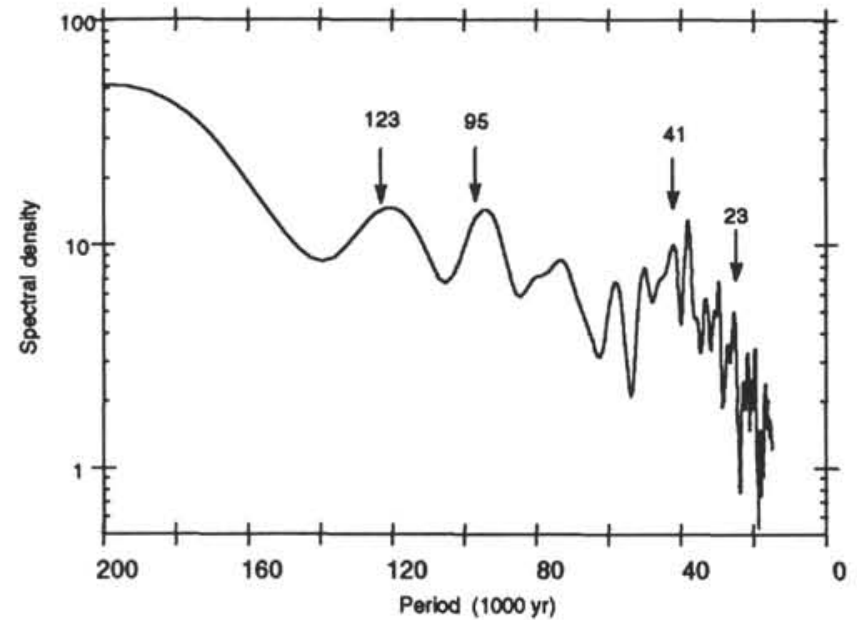

Figure 10. Spectral analysis of the SFLU section from 3.5 to $8.75 \mathrm{Ma}$ in Hole 806B. The time scale is based on shipboard analyses. Split 41 and 23 k.y. periods point to the need for minor tuning of the sedimentation rates.

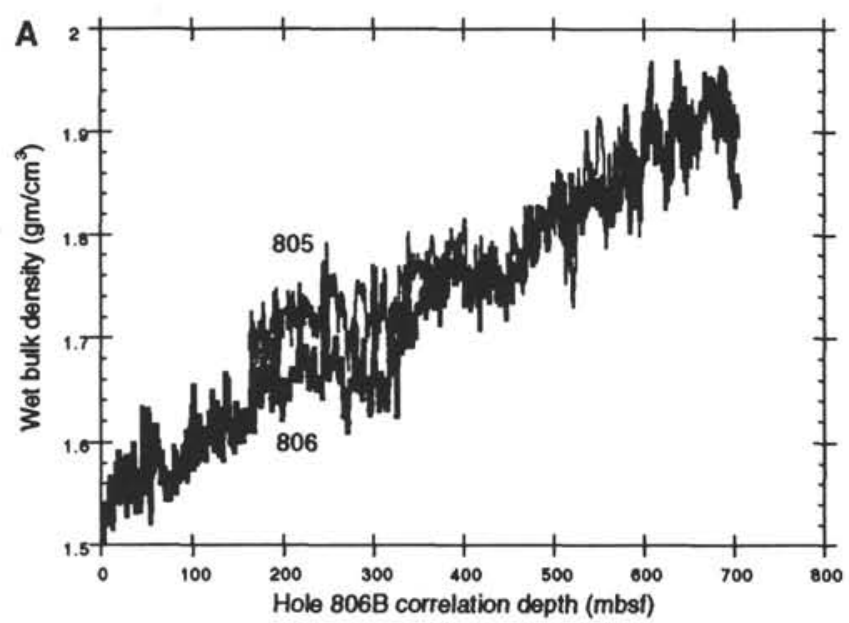

B

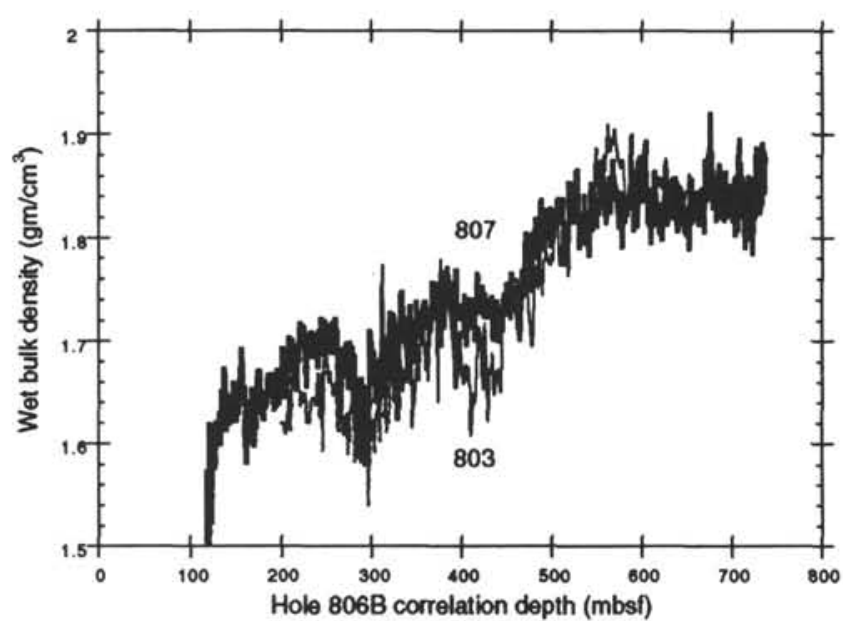

Figure 11. Correlation between Sites 806 and 805 (A) and Sites 807 and 803 (B). All depths are plotted on the reference Site 806 depth scale.

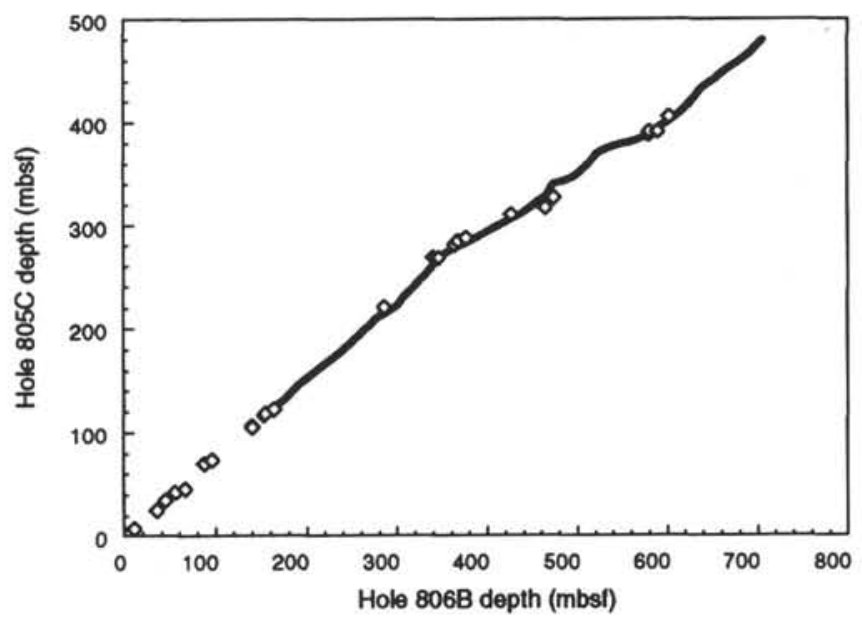

Figure 12. Stratigraphic correlation between Holes $806 \mathrm{~B}$ and $805 \mathrm{C}$, showing the inverse signal correlation map (solid line) compared to the nannofossil biostratigraphic events identified from the detailed post-cruise analysis (Takayama and Backman, this volume).

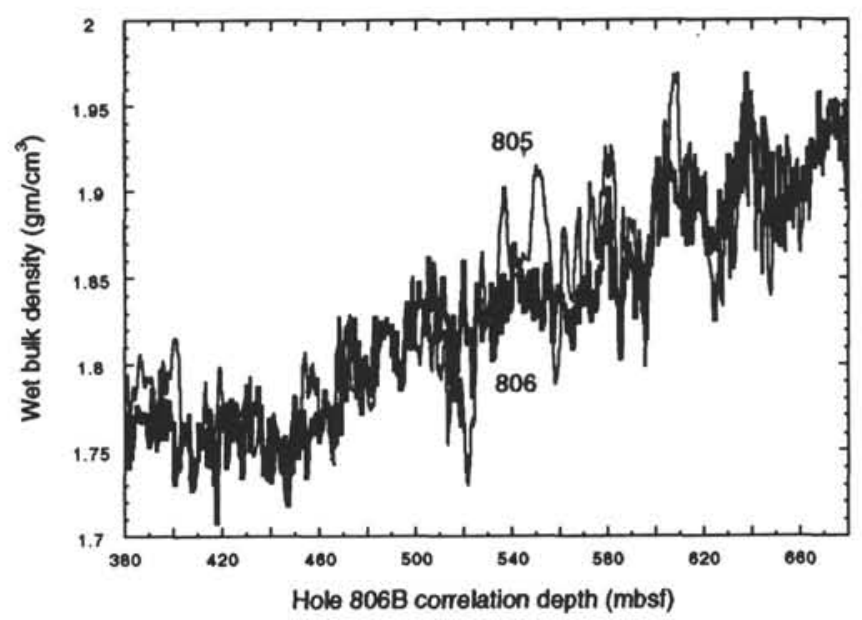

Figure 13. Detail of correlation between Holes $806 \mathrm{~B}$ and $805 \mathrm{C}$ over the "kinky" interval in Figure 12.

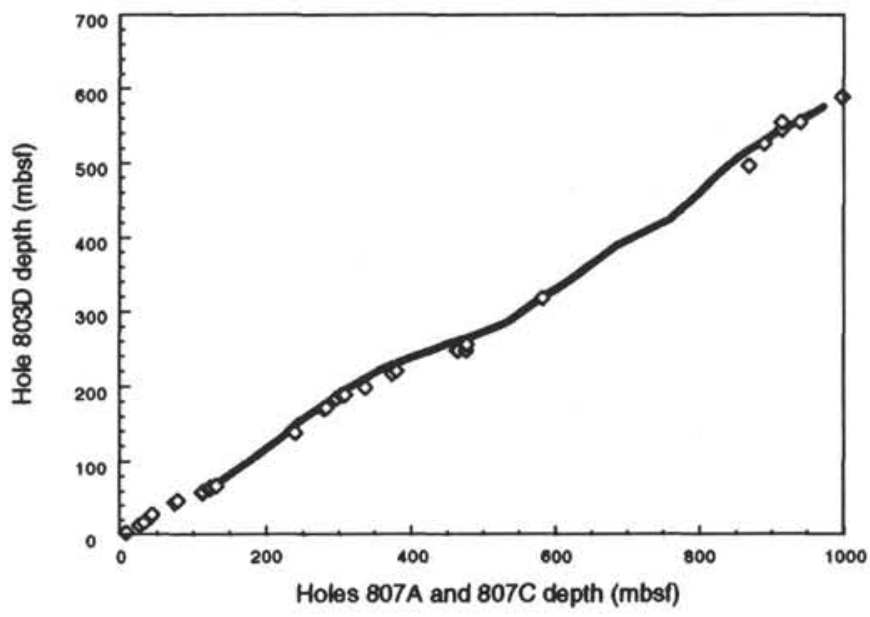

Figure 14. Stratigraphic correlation between Site 807 and Hole 803D, showing the inverse signal correlation map (solid line) compared to the nannofossil biostratigraphic events identified from the detailed post-cruise analysis (Takayama and Backman, this volume). 


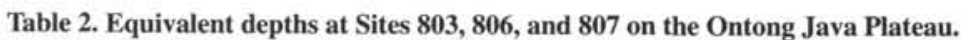

\begin{tabular}{|c|c|c|c|c|c|c|c|c|c|c|c|c|c|c|}
\hline $\begin{array}{l}\text { Hole } \\
807 \mathrm{~A}\end{array}$ & $\begin{array}{l}\text { Hole } \\
807 \mathrm{C}\end{array}$ & Site 807 & $\begin{array}{l}\text { Hole } \\
806 \mathrm{~B}\end{array}$ & $\begin{array}{l}\text { Hole } \\
803 \mathrm{D}\end{array}$ & $\begin{array}{l}\text { Hole } \\
807 A\end{array}$ & $\begin{array}{l}\text { Hole } \\
807 \mathrm{C}\end{array}$ & Site 807 & $\begin{array}{l}\text { Hole } \\
806 \mathrm{~B}\end{array}$ & $\begin{array}{l}\text { Hole } \\
803 \mathrm{D}\end{array}$ & $\begin{array}{l}\text { Hole } \\
807 \mathrm{~A}\end{array}$ & $\begin{array}{l}\text { Hole } \\
807 \mathrm{C}\end{array}$ & Site 807 & $\begin{array}{l}\text { Hole } \\
806 \mathrm{~B}\end{array}$ & $\begin{array}{r}\text { Hole } \\
803 \mathrm{D}\end{array}$ \\
\hline $\begin{array}{l}\text { depth } \\
\text { (mbsf) }\end{array}$ & $\begin{array}{l}\text { depth } \\
\text { (mbsf) }\end{array}$ & $\begin{array}{l}\text { age } \\
(\mathrm{Ma})\end{array}$ & $\begin{array}{l}\text { depth } \\
\text { (mbsf) }\end{array}$ & $\begin{array}{c}\text { depth } \\
\text { (mbsf) }\end{array}$ & $\begin{array}{l}\text { depth } \\
\text { (mbsf) }\end{array}$ & $\begin{array}{l}\text { depth } \\
\text { (mbsf) }\end{array}$ & $\begin{array}{c}\text { age } \\
(\mathrm{Ma})\end{array}$ & $\begin{array}{c}\text { depth } \\
\text { (mbsf) }\end{array}$ & $\begin{array}{c}\text { depth } \\
\text { (mbsf) }\end{array}$ & $\begin{array}{l}\text { depth } \\
\text { (mbsf) }\end{array}$ & $\begin{array}{c}\text { depth } \\
\text { (mbsf) }\end{array}$ & $\begin{array}{c}\text { age } \\
(\mathrm{Ma})\end{array}$ & $\begin{array}{c}\text { depth } \\
\text { (mbsf) }\end{array}$ & $\begin{array}{l}\text { depth } \\
\text { (mbsf) }\end{array}$ \\
\hline 139.0 & & 5.308 & 167.87 & 73.79 & 215.0 & & 7.019 & 263.43 & 127.15 & 291.0 & & 9.364 & 349.42 & 182.99 \\
\hline 140.0 & & 5.330 & 169.15 & 74.48 & 216.0 & & 7.041 & 264.62 & 127.85 & 292.0 & & 9.449 & 350.71 & 183.68 \\
\hline 141.0 & & 5.353 & 170.44 & 75.17 & 217.0 & & 7.064 & 265.80 & 128.54 & 293.0 & & 9.535 & 352.01 & 184.38 \\
\hline 142.0 & & 5.375 & 171.73 & 75.86 & 218.0 & & 7.086 & 266.98 & 129.23 & 294.0 & & 9.620 & 353.34 & 185.07 \\
\hline 143.0 & & 5.398 & 172.97 & 76.56 & 219.0 & & 7.109 & 268.15 & 129.93 & 295.0 & & 9.705 & 354.65 & 185.76 \\
\hline 144.0 & & 5.420 & 174.11 & 77.24 & 220.0 & & 7.131 & 269.32 & 130.81 & 296.0 & & 9.790 & 355.99 & 186.45 \\
\hline 145.0 & & 5.443 & 175.26 & 77.94 & 221.0 & & 7.154 & 270.50 & 131.71 & 297.0 & & 9.875 & 357.32 & 187.14 \\
\hline 146.0 & & 5.465 & 176.41 & 78.63 & 222.0 & & 7.176 & 271.65 & 132.61 & 298.0 & & 9.960 & 358.65 & 187.83 \\
\hline 147.0 & & 5.488 & 177.57 & 79.32 & 223.0 & & 7.199 & 272.79 & 133.50 & 299.0 & & 10.045 & 359.98 & 188.53 \\
\hline 148.0 & & 5.510 & 178.73 & 80.01 & 224.0 & & 7.221 & 273.89 & 134.40 & 300.0 & & 10.130 & 361.27 & 189.22 \\
\hline 149.0 & & 5.533 & 179.88 & 80.70 & 225.0 & & 7.244 & 274.97 & 135.29 & 301.0 & & 10.215 & 362.58 & 189.91 \\
\hline 150.0 & & 5.555 & 181.05 & 81.39 & 226.0 & & 7.266 & 276.06 & 136.19 & 302.0 & & 10.300 & 363.85 & 190.60 \\
\hline 151.0 & & 5.578 & 182.21 & 82.08 & 227.0 & & 7.289 & 277.12 & 137.08 & 303.0 & & 10.385 & 365.12 & 191.29 \\
\hline 152.0 & & 5.600 & 183.36 & 82.77 & 228.0 & & 7.311 & 278.19 & 137.97 & 304.0 & & 10.470 & 366.35 & 191.99 \\
\hline 153.0 & & 5.623 & 184.52 & 83.46 & 229.0 & & 7.334 & 279.23 & 138.87 & 305.0 & & 10.555 & 367.60 & 192.67 \\
\hline 154.0 & & 5.645 & 185.68 & 84.15 & 230.0 & & 7.356 & 280.31 & 139.76 & 306.0 & & 10.640 & 369.04 & 193.37 \\
\hline 155.0 & & 5.668 & 187.06 & 84.84 & 231.0 & & 7.379 & 281.36 & 140.64 & 307.0 & & 10.725 & 370.47 & 194.06 \\
\hline 156.0 & & 5.690 & 188.43 & 85.54 & 232.0 & & 7.401 & 282.39 & 141.54 & 308.0 & & 10.810 & 371.91 & 194.74 \\
\hline 157.0 & & 5.713 & 189.78 & 86.23 & 233.0 & & 7.424 & 283.43 & 142.42 & 309.0 & & 10.896 & 373.31 & 195.43 \\
\hline 158.0 & & 5.735 & 191.13 & 86.92 & 234.0 & & 7.446 & 284.43 & 143.31 & 310.0 & & 10.981 & 374.71 & 196.03 \\
\hline 159.0 & & 5.758 & 192.43 & 87.61 & 235.0 & & 7.469 & 285.43 & 144.20 & 311.0 & & 11.066 & 376.11 & 196.52 \\
\hline 160.0 & & 5.781 & 193.70 & 88.31 & 236.0 & & 7.491 & 286.45 & 145.09 & 312.0 & & 11.151 & 377.50 & 197.05 \\
\hline 161.0 & & 5.803 & 194.94 & 89.00 & 237.0 & & 7.514 & 287.53 & 145.97 & 313.0 & & 11.236 & 378.91 & 197.57 \\
\hline 162.0 & & 5.826 & 196.19 & 89.69 & 238.0 & & 7.536 & 288.60 & 146.87 & 314.0 & & 11.321 & 380.31 & 198.09 \\
\hline 163.0 & & 5.848 & 197.41 & 90.39 & 239.0 & & 7.559 & 289.67 & 147.76 & 315.0 & & 11.406 & 381.73 & 198.61 \\
\hline 164.0 & & 5.871 & 198.66 & 91.08 & 240.0 & & 7.586 & 290.74 & 148.65 & 316.0 & & 11.491 & 383.14 & 199.14 \\
\hline 165.0 & & 5.893 & 199.88 & 91.78 & 241.0 & & 7.613 & 291.78 & 149.54 & 317.0 & & 11.521 & 384.56 & 199.66 \\
\hline 166.0 & & 5.916 & 201.10 & 92.48 & 242.0 & & 7.640 & 292.83 & 150.44 & 318.0 & & 11.550 & 385.98 & 200.18 \\
\hline 167.0 & & 5.938 & 202.34 & 93.17 & 243.0 & & 7.667 & 293.87 & 151.33 & 319.0 & & 11.580 & 387.41 & 200.70 \\
\hline 168.0 & & 5.961 & 203.55 & 93.87 & 244.0 & & 7.693 & 294.92 & 152.22 & 320.0 & & 11.609 & 388.86 & 201.22 \\
\hline 169.0 & & 5.983 & 204.82 & 94.57 & 245.0 & & 7.720 & 295.96 & 153.06 & 321.0 & & 11.639 & 390.28 & 201.74 \\
\hline 170.0 & & 6.006 & 206.08 & 95.27 & 246.0 & & 7.747 & 297.02 & 153.66 & 322.0 & & 11.669 & 391.73 & 202.26 \\
\hline 171.0 & & 6.028 & 207.35 & 95.97 & 247.0 & & 7.774 & 298.10 & 154.27 & 323.0 & & 11.698 & 393.15 & 202.78 \\
\hline 172.0 & & 6.051 & 208.63 & 96.67 & 248.0 & & 7.801 & 299.22 & 154.88 & 324.0 & & 11.728 & 394.57 & 203.30 \\
\hline 173.0 & & 6.073 & 209.90 & 97.37 & 249.0 & & 7.828 & 300.43 & 155.49 & 325.0 & & 11.757 & 395.99 & 203.81 \\
\hline 174.0 & & 6.096 & 211.18 & 98.07 & 250.0 & & 7.855 & 301.66 & 156.10 & 326.0 & & 11.787 & 397.39 & 204.33 \\
\hline 175.0 & & 6.118 & 212.45 & 98.77 & 251.0 & & 7.882 & 302.70 & 156.72 & 327.0 & & 11.817 & 398.75 & 204.84 \\
\hline 176.0 & & 6.141 & 213.68 & 99.47 & 252.0 & & 7.909 & 303.77 & 157.33 & 328.0 & & 11.846 & 399.83 & 205.35 \\
\hline 177.0 & & 6.163 & 214.92 & 100.17 & 253.0 & & 7.935 & 304.85 & 157.95 & 329.0 & & 11.876 & 400.98 & 205.87 \\
\hline 178.0 & & 6.186 & 216.17 & 100.87 & 254.0 & & 7.962 & 305.98 & 158.57 & 330.0 & & 11.905 & 402.12 & 206.38 \\
\hline 179.0 & & 6.208 & 217.43 & 101.59 & 255.0 & & 7.989 & 307.12 & 159.19 & 331.0 & & 11.935 & 403.27 & 206.89 \\
\hline 180.0 & & 6.231 & 218.68 & 102.30 & 256.0 & & 8.016 & 308.30 & 159.82 & 332.0 & & 11.965 & 404.42 & 207.40 \\
\hline 181.0 & & 6.253 & 219.95 & 103.02 & 257.0 & & 8.043 & 309.49 & 160.44 & 333.0 & & 11.994 & 405.57 & 207.91 \\
\hline 182.0 & & 6.276 & 221.21 & 103.74 & 258.0 & & 8.070 & 310.73 & 161.07 & 334.0 & & 12.024 & 406.72 & 208.42 \\
\hline 183.0 & & 6.298 & 222.48 & 104.46 & 259.0 & & 8.097 & 311.99 & 161.70 & 335.0 & & 12.053 & 407.89 & 208.93 \\
\hline 184.0 & & 6.321 & 223.76 & 105.17 & 260.0 & & 8.124 & 313.12 & 162.33 & 336.0 & & 12.083 & 409.14 & 209.44 \\
\hline 185.0 & & 6.343 & 225.04 & 105.89 & 261.0 & & 8.151 & 314.22 & 162.97 & 337.0 & & 12.113 & 410.55 & 209.95 \\
\hline 186.0 & & 6.366 & 226.33 & 106.61 & 262.0 & & 8.177 & 315.33 & 163.60 & 338.0 & & 12.142 & 411.99 & 210.46 \\
\hline 187.0 & & 6.388 & 227.61 & 107.32 & 263.0 & & 8.204 & 316.45 & 164.24 & 339.0 & & 12.172 & 413.46 & 211.02 \\
\hline 188.0 & & 6.411 & 228.92 & 108.04 & 264.0 & & 8.231 & 317.60 & 164.88 & 340.0 & & 12.201 & 414.95 & 211.59 \\
\hline 189.0 & & 6.433 & 230.21 & 108.76 & 265.0 & & 8.258 & 318.74 & 165.53 & 341.0 & & 12.231 & 416.48 & 212.16 \\
\hline 190.0 & & 6.456 & 231.51 & 109.47 & 266.0 & & 8.285 & 319.89 & 166.18 & 342.0 & & 12.261 & 418.00 & 212.73 \\
\hline 191.0 & & 6.478 & 232.82 & 110.19 & 267.0 & & 8.312 & 321.03 & 166.83 & 343.0 & & 12.290 & 419.52 & 213.30 \\
\hline 192.0 & & 6.501 & 234.13 & 110.91 & 268.0 & & 8.339 & 322.19 & 167.47 & 344.0 & & 12.320 & 421.04 & 213.87 \\
\hline 193.0 & & 6.523 & 235.46 & 111.62 & 269.0 & & 8.366 & 323.32 & 168.13 & 345.0 & & 12.349 & 422.57 & 214.43 \\
\hline 194.0 & & 6.546 & 236.77 & 112.34 & 270.0 & & 8.393 & 324.63 & 168.78 & 346.0 & & 12.379 & 424.10 & 215.00 \\
\hline 195.0 & & 6.568 & 238.10 & 113.05 & 271.0 & & 8.419 & 325.93 & 169.44 & 347.0 & & 12.409 & 425.62 & 215.56 \\
\hline 196.0 & & 6.591 & 239.34 & 113.76 & 272.0 & & 8.446 & 327.21 & 170.10 & 348.0 & & 12.438 & 427.12 & 216.12 \\
\hline 197.0 & & 6.613 & 240.60 & 114.48 & 273.0 & & 8.473 & 328.48 & 170.76 & 349.0 & & 12.468 & 428.58 & 216.69 \\
\hline 198.0 & & 6.636 & 241.86 & 115.19 & 274.0 & & 8.500 & 329.71 & 171.43 & 350.0 & & 12.497 & 430.02 & 217.25 \\
\hline 199.0 & & 6.658 & 243.13 & 115.90 & 275.0 & & 8.527 & 330.93 & 172.10 & 351.0 & & 12.527 & 431.42 & 217.81 \\
\hline 200.0 & & 6.681 & 244.40 & 116.61 & 276.0 & & 8.554 & 332.11 & 172.76 & 352.0 & & 12.557 & 432.78 & 218.37 \\
\hline 201.0 & & 6.704 & 245.66 & 117.32 & 277.0 & & 8.581 & 333.30 & 173.44 & 353.0 & & 12.586 & 434.09 & 218.92 \\
\hline 202.0 & & 6.726 & 246.95 & 118.04 & 278.0 & & 8.608 & 334.46 & 174.11 & 354.0 & & 12.616 & 435.36 & 219.48 \\
\hline 203.0 & & 6.749 & 248.22 & 118.74 & 279.0 & & 8.635 & 335.61 & 174.78 & 355.0 & & 12.645 & 436.61 & 220.03 \\
\hline 204.0 & & 6.771 & 249.51 & 119.45 & 280.0 & & 8.661 & 336.75 & 175.46 & 356.0 & & 12.675 & 437.81 & 220.58 \\
\hline 205.0 & & 6.794 & 250.79 & 120.16 & 281.0 & & 8.688 & 337.88 & 176.14 & 357.0 & 355.55 & 12.705 & 438.99 & 221.10 \\
\hline 206.0 & & 6.816 & 252.07 & 120.86 & 282.0 & & 8.715 & 339.02 & 176.82 & 358.0 & 356.55 & 12.734 & 440.15 & 221.50 \\
\hline 207.0 & & 6.839 & 253.40 & 121.56 & 283.0 & & 8.742 & 340.15 & 177.50 & 359.0 & 357.54 & 12.764 & 441.28 & 221.90 \\
\hline 208.0 & & 6.861 & 254.95 & 122.26 & 284.0 & & 8.769 & 341.26 & 178.18 & 360.0 & 358.54 & 12.793 & 442.41 & 222.30 \\
\hline 209.0 & & 6.884 & 256.41 & 122.97 & 285.0 & & 8.854 & 342.29 & 178.87 & 361.0 & 359.54 & 12.823 & 443.52 & 222.70 \\
\hline 210.0 & & 6.906 & 257.56 & 123.67 & 286.0 & & 8.939 & 343.33 & 179.55 & 362.0 & 360.55 & 12.853 & 444.64 & 223.10 \\
\hline 211.0 & & 6.929 & 258.73 & 124.37 & 287.0 & & 9.024 & 344.41 & 180.24 & 363.0 & 361.57 & 12.882 & 445.76 & 223.50 \\
\hline 212.0 & & 6.951 & 259.89 & 125.07 & 288.0 & & 9.109 & 345.63 & 180.92 & 364.0 & 362.60 & 12.912 & 446.89 & 223.89 \\
\hline 213.0 & & 6.974 & 261.06 & 125.76 & 289.0 & & 9.194 & 346.88 & 181.61 & 365.0 & 363.63 & 12.941 & 448.11 & 224.29 \\
\hline 214.0 & & 6.996 & 262.25 & 126.46 & 290.0 & & 9.279 & 348.14 & 182.30 & 366.0 & 364.68 & 12.971 & 449.36 & 224.68 \\
\hline
\end{tabular}


Table 2 (continued).

\begin{tabular}{|c|c|c|c|c|c|c|c|c|c|c|c|c|c|c|}
\hline $\begin{array}{r}\text { Hole } \\
807 \mathrm{~A}\end{array}$ & $\begin{array}{l}\text { Hole } \\
807 \mathrm{C}\end{array}$ & Site 807 & $\begin{array}{l}\text { Hole } \\
806 \mathrm{~B}\end{array}$ & $\begin{array}{l}\text { Hole } \\
\text { 803D }\end{array}$ & $\begin{array}{r}\text { Hole } \\
807 \mathrm{~A}\end{array}$ & $\begin{array}{l}\text { Hole } \\
807 \mathrm{C}\end{array}$ & Site 807 & $\begin{array}{l}\text { Hole } \\
806 \mathrm{~B}\end{array}$ & $\begin{array}{l}\text { Hole } \\
\text { 803D }\end{array}$ & $\begin{array}{l}\text { Hole } \\
807 \mathrm{~A}\end{array}$ & $\begin{array}{l}\text { Hole } \\
807 \mathrm{C}\end{array}$ & Site 807 & $\begin{array}{c}\text { Hole } \\
806 \mathrm{~B}\end{array}$ & $\begin{array}{r}\text { Hole } \\
803 \mathrm{D}\end{array}$ \\
\hline $\begin{array}{c}\text { depth } \\
\text { (mbsf) }\end{array}$ & $\begin{array}{c}\text { depth } \\
\text { (mbsf) }\end{array}$ & $\begin{array}{l}\text { age } \\
\text { (Ma) }\end{array}$ & $\begin{array}{c}\text { depth } \\
\text { (mbsf) }\end{array}$ & $\begin{array}{r}\text { depth } \\
\text { (mbsf) }\end{array}$ & $\begin{array}{c}\text { depth } \\
\text { (mbsf) }\end{array}$ & $\begin{array}{l}\text { depth } \\
\text { (mbsf) }\end{array}$ & $\begin{array}{c}\text { age } \\
\text { (Ma) }\end{array}$ & $\begin{array}{c}\text { depth } \\
\text { (mbsf) }\end{array}$ & $\begin{array}{c}\text { depth } \\
\text { (mbsf) }\end{array}$ & $\begin{array}{c}\text { depth } \\
\text { (mbsf) }\end{array}$ & $\begin{array}{c}\text { depth } \\
\text { (mbsf) }\end{array}$ & $\begin{array}{l}\text { age } \\
\text { (Ma) }\end{array}$ & $\begin{array}{c}\text { depth } \\
\text { (mbsf) }\end{array}$ & $\begin{array}{r}\text { depth } \\
\text { (mbsf) }\end{array}$ \\
\hline 367.0 & 365.73 & 13.001 & 450.67 & 225.07 & 442.0 & 440.86 & 17.293 & 554.22 & 252.73 & 517.0 & 516.47 & 21.680 & 658.21 & 278.29 \\
\hline 368.0 & 366.79 & 13.030 & 451.99 & 225.46 & 443.0 & 441.90 & 17.366 & 555.67 & 253.12 & 518.0 & 517.47 & 21.721 & 659.76 & 278.67 \\
\hline 369.0 & 367.86 & 13.060 & 453.36 & 225.85 & 444.0 & 442.93 & 17.439 & 557.13 & 253.59 & 519.0 & 518.47 & 21.761 & 661.31 & 279.06 \\
\hline 370.0 & 368.94 & 13.089 & 454.77 & 226.24 & 445.0 & 443.98 & 17.512 & 558.57 & 254.06 & 520.0 & 519.48 & 21.801 & 662,88 & 279.45 \\
\hline 371.0 & 370.02 & 13.119 & 456.23 & 226.62 & 446.0 & 445.03 & 17.584 & 559.99 & 254.53 & 521.0 & 520.48 & 21.842 & 664.18 & 279.85 \\
\hline 372.0 & 371.10 & 13.149 & 457.74 & 227.01 & 447.0 & 446.08 & 17.657 & 561.40 & 255.01 & 522.0 & 521.48 & 21.882 & 665.34 & 280.24 \\
\hline 373.0 & 372.18 & 13.178 & 459.27 & 227.39 & 448.0 & 447.13 & 17.730 & 562.82 & 255.48 & 523.0 & 522.48 & 21.922 & 666.50 & 280.64 \\
\hline 374.0 & 373.26 & 13.208 & 460.85 & 227.78 & 449.0 & 448.18 & 17.803 & 564.25 & 255.95 & 524.0 & 523.48 & 21.963 & 667.66 & 281.04 \\
\hline 375.0 & 374.34 & 13.237 & 462.44 & 228.15 & 450.0 & 449.23 & 17.875 & 565.57 & 256.33 & 525.0 & 524.49 & 22.003 & 668.83 & 281.44 \\
\hline 376.0 & 375.41 & 13.267 & 464.04 & 228.54 & 451.0 & 450.28 & 17.948 & 566.84 & 256.59 & 526.0 & 525.49 & 22.043 & 669.99 & 281.85 \\
\hline 377.0 & 376.47 & 13.297 & 465.55 & 228.91 & 452.0 & 451.33 & 18.021 & 568.11 & 256.87 & 527.0 & 526.49 & 22.084 & 671.17 & 282.25 \\
\hline 378.0 & 377.53 & 13.326 & 466.99 & 229.29 & 453.0 & 452.37 & 18.094 & 569.38 & 257.16 & 528.0 & 527.49 & 22.124 & 672.18 & 282.66 \\
\hline 379.0 & 378.57 & 13.356 & 468.40 & 229.67 & 454.0 & 453.41 & 18.167 & 570.66 & 257.44 & 529.0 & 528.50 & 22.164 & 673.07 & 283.07 \\
\hline 380.0 & 379.60 & 13.385 & 469.77 & 230.04 & 455.0 & 454.45 & 18.239 & 572.18 & 257.72 & 530.0 & 529.50 & 22.205 & 673.95 & 283.48 \\
\hline 381.0 & 380.62 & 13.415 & 471.11 & 230.41 & 456.0 & 455.48 & 18.312 & 574.09 & 258.01 & 531.0 & 530.50 & 22.245 & 674.86 & 283.90 \\
\hline 382.0 & 381.63 & 13.445 & 472.39 & 230.79 & 457.0 & 456.51 & 18.385 & 576.00 & 258.29 & 532.0 & 531.49 & 22.285 & 675.74 & 284.30 \\
\hline 383.0 & 382.63 & 13.474 & 473.66 & 231.16 & 458.0 & 457.53 & 18.458 & 577.93 & 258.58 & 533.0 & 532.49 & 22.326 & 676.70 & 284.98 \\
\hline 384.0 & 383.61 & 13.504 & 474.88 & 231.53 & 459.0 & 458.55 & 18.530 & 579.83 & 258.86 & 534.0 & 533.48 & 22.366 & 677.69 & 285.66 \\
\hline 385.0 & 384.59 & 13.533 & 476.10 & 231.89 & 460.0 & 459.57 & 18.603 & 581.75 & 259.14 & 535.0 & 534.48 & 22.406 & 678.68 & 286.34 \\
\hline 386.0 & 385.55 & 13.563 & 477.29 & 232.26 & 461.0 & 460.58 & 18.676 & 583.67 & 259.42 & 536.0 & 535.47 & 22.446 & 679.67 & 287.03 \\
\hline 387.0 & 386.51 & 13.593 & 478.46 & 232.63 & 462.0 & 461.59 & 18.749 & 585.23 & 259.71 & 537.0 & 536.46 & 22.487 & 680.65 & 287.72 \\
\hline 388.0 & 387.47 & 13.622 & 479.58 & 232.99 & 463.0 & 462.59 & 18.822 & 586.22 & 259.99 & 538.0 & 537.44 & 22.527 & 681.63 & 288.40 \\
\hline 389.0 & 388.42 & 13.652 & 480.54 & 233.35 & 464.0 & 463.60 & 18.894 & 587.20 & 260.27 & 539.0 & 538.43 & 22.567 & 682.62 & 289.09 \\
\hline 390.0 & 389.36 & 13.681 & 481.51 & 233.72 & 465.0 & 464.59 & 18.967 & 588.17 & 260.55 & 540.0 & 539.41 & 22.608 & 683.60 & 289.79 \\
\hline 391.0 & 390.31 & 13.711 & 482.48 & 234.08 & 466.0 & 465.59 & 19.040 & 589.16 & 260.83 & 541.0 & 540.39 & 22.648 & 684.59 & 290.48 \\
\hline 392.0 & 391.26 & 13.741 & 483.47 & 234.44 & 467.0 & 466.58 & 19.113 & 590.13 & 261.11 & 542.0 & 541.37 & 22.688 & 685.56 & 291.17 \\
\hline 393.0 & 392.21 & 13.770 & 484.48 & 234.80 & 468.0 & 467.57 & 19.185 & 591.12 & 261.39 & 543.0 & 542.35 & 22.729 & 686.57 & 291.87 \\
\hline 394.0 & 393.17 & 13.800 & 485.49 & 235.16 & 469.0 & 468.56 & 19.258 & 592.09 & 261.67 & 544.0 & 543.33 & 22.769 & 687.62 & 292.56 \\
\hline 395.0 & 394.13 & 13.873 & 486.54 & 235.52 & 470.0 & 469.55 & 19.331 & 593.07 & 261.95 & 545.0 & 544.30 & 22.809 & 688.66 & 293.26 \\
\hline 396.0 & 395.10 & 13.945 & 487.60 & 235.87 & 471.0 & 470.53 & 19.404 & 594.06 & 262.22 & 546.0 & 545.28 & 22.850 & 689.72 & 293.95 \\
\hline 397.0 & 396.08 & 14.018 & 488.70 & 236.23 & 472.0 & 471.52 & 19.477 & 595.03 & 262.50 & 547.0 & 546.26 & 22.890 & 690.75 & 294.65 \\
\hline 398.0 & 397.07 & 14.091 & 489.83 & 236.59 & 473.0 & 472.50 & 19.549 & 596.02 & 262.77 & 548.0 & 547.24 & 22.930 & 691.80 & 295.35 \\
\hline 399.0 & 398.08 & 14.164 & 491.00 & 236.94 & 474.0 & 473.48 & 19.622 & 596.99 & 263.05 & 549.0 & 548.22 & 22.971 & 692.83 & 296.04 \\
\hline 400.0 & 399.09 & 14.237 & 492.35 & 237.30 & 475.0 & 474.46 & 19.695 & 597.98 & 263.32 & 550.0 & 549.21 & 23.011 & 693.88 & 296.74 \\
\hline 401.0 & 400.11 & 14.309 & 493.83 & 237.65 & 476.0 & 475.44 & 19.768 & 598.95 & 263.60 & 551.0 & 550.19 & 23.051 & 694.90 & 297.43 \\
\hline 402.0 & 401.15 & 14.382 & 495.37 & 238.01 & 477.0 & 476.43 & 19.840 & 600.22 & 263.87 & 552.0 & 551.17 & 23.092 & 695.93 & 298.13 \\
\hline 403.0 & 402.20 & 14.455 & 496.93 & 238.36 & 478.0 & 477.41 & 19.913 & 601.69 & 264.14 & 553.0 & 552.16 & 23.132 & 696.95 & 298.82 \\
\hline 404.0 & 403.25 & 14.528 & 498.53 & 238.72 & 479.0 & 478.39 & 19.986 & 603.15 & 264.43 & 554.0 & 553.15 & 23.172 & 698.13 & 299.51 \\
\hline 405.0 & 404.31 & 14.600 & 499.88 & 239.07 & 480.0 & 479.38 & 20.059 & 604.62 & 264.79 & 555.0 & 554.14 & 23.213 & 699.33 & 300.20 \\
\hline 406.0 & 405.38 & 14.673 & 501.16 & 239.43 & 481.0 & 480.36 & 20.132 & 606.08 & 265.15 & 556.0 & 555.14 & 23.253 & 700.38 & 300.89 \\
\hline 407.0 & 406.45 & 14.746 & 502.46 & 239.78 & 482.0 & 481.35 & 20.204 & 607.46 & 265.51 & 557.0 & 556.13 & 23.293 & 701.29 & 301.57 \\
\hline 408.0 & 407.53 & 14.819 & 503.76 & 240.14 & 483.0 & 482.33 & 20.277 & 608.76 & 265.87 & 558.0 & 557.13 & 23.334 & 702.20 & 302.25 \\
\hline 409.0 & 408.60 & 14.892 & 505.11 & 240.49 & 484.0 & 483.32 & 20.350 & 610.09 & 266.23 & 559.0 & 558.12 & 23.374 & 703.09 & 302.93 \\
\hline 410.0 & 409.67 & 14.964 & 506.57 & 240.85 & 485.0 & 484.31 & 20.390 & 611.40 & 266.59 & 560.0 & 559.12 & 23.414 & 704.00 & 303.61 \\
\hline 411.0 & 410.73 & 15.037 & 508.56 & 241.20 & 486.0 & 485.30 & 20.430 & 612.70 & 266.95 & 561.0 & 560.11 & 23.454 & 704.89 & 304.29 \\
\hline 412.0 & 411.79 & 15.110 & 510.54 & 241.56 & 487.0 & 486.30 & 20.471 & 614.01 & 267.31 & 562.0 & 561.11 & 23.495 & 705.80 & 304.96 \\
\hline 413.0 & 412.83 & 15.183 & 512.51 & 241.92 & 488.0 & 487.29 & 20.511 & 615.29 & 267.66 & 563.0 & 562.10 & 23.535 & 706.70 & 305.64 \\
\hline 414.0 & 413.87 & 15.255 & 514.49 & 242.27 & 489.0 & 488.29 & 20.551 & 616.59 & 268.02 & 564.0 & 563.09 & 23.575 & 707.60 & 306.30 \\
\hline 415.0 & 414.89 & 15.328 & 516.45 & 242.64 & 490.0 & 489.29 & 20.592 & 617.86 & 268.38 & 565.0 & 564.09 & 23.616 & 708.50 & 306.97 \\
\hline 416.0 & 415.90 & 15.401 & 518.42 & 242.99 & 491.0 & 490.29 & 20.632 & 619.14 & 268.74 & 566.0 & 565.08 & 23.656 & 709.40 & 307.63 \\
\hline 417.0 & 416.90 & 15.474 & 520.37 & 243.35 & 492.0 & 491.29 & 20.672 & 620.41 & 269.09 & 567.0 & 566.06 & 23.696 & 710.31 & 308.29 \\
\hline 418.0 & 417.89 & 15.547 & 522.16 & 243.71 & 493.0 & 492.30 & 20.713 & 621.66 & 269.45 & 568.0 & 567.05 & 23.737 & 711.21 & 308.95 \\
\hline 419.0 & 418.86 & 15.619 & 523.43 & 244.08 & 494.0 & 493.31 & 20.753 & 622.91 & 269.81 & 569.0 & 568.03 & 23.777 & 712.12 & 309.60 \\
\hline 420.0 & 419.82 & 15.692 & 524.67 & 244.44 & 495.0 & 494.32 & 20.793 & 624.14 & 270.17 & 570.0 & 569.01 & 23.817 & 713.02 & 310.25 \\
\hline 421.0 & 420.78 & 15.765 & 525.91 & 244.80 & 496.0 & 495.33 & 20.834 & 625.37 & 270.52 & 571.0 & 569.99 & 23.858 & 713.93 & 310.90 \\
\hline 422.0 & 421.72 & 15.838 & 527.15 & 245.17 & 497.0 & 496.34 & 20.874 & 626.59 & 270.88 & 572.0 & 570.97 & 23.898 & 714.84 & 311.54 \\
\hline 423.0 & 422.66 & 15.910 & 528.40 & 245.53 & 498.0 & 497.35 & 20.914 & 627.80 & 271.24 & 573.0 & 571.94 & 23.938 & 715.75 & 312.18 \\
\hline 424.0 & 423.59 & 15.983 & 529.65 & 245.90 & 499.0 & 498.36 & 20.955 & 629.00 & 271.60 & 574.0 & 572.92 & 23.979 & 716.78 & 312.82 \\
\hline 425.0 & 424.52 & 16.056 & 530.91 & 246.27 & 500.0 & 499.38 & 20.995 & 630.20 & 271.96 & 575.0 & 573.89 & 24.019 & 717.92 & 313.57 \\
\hline 426.0 & 425.44 & 16.129 & 532.15 & 246.64 & 501.0 & 500.39 & 21.035 & 631.39 & 272.32 & 576.0 & 574.86 & 24.059 & 719.01 & 314.22 \\
\hline 427.0 & 426.36 & 16.202 & 533.41 & 247.01 & 502.0 & 501.40 & 21.076 & 632.55 & 272.68 & 577.0 & 575.83 & 24.100 & 720.05 & 314.86 \\
\hline 428.0 & 427.28 & 16.274 & 534.66 & 247.38 & 503.0 & 502.42 & 21.116 & 633.72 & 273.05 & 578.0 & 576.81 & 24.140 & 721.10 & 315.50 \\
\hline 429.0 & 428.21 & 16.347 & 535.92 & 247.76 & 504.0 & 503.43 & 21.156 & 634.89 & 273.41 & 579.0 & 577.78 & 24.180 & 722.15 & 316.13 \\
\hline 430.0 & 429.14 & 16.420 & 537.28 & 248.13 & 505.0 & 504.44 & 21.197 & 636.04 & 273.78 & 580.0 & 578.75 & 24.221 & 723.20 & 316.76 \\
\hline 431.0 & 430.07 & 16.493 & 538.66 & 248.51 & 506.0 & 505.45 & 21.237 & 637.82 & 274.15 & 581.0 & 579.73 & 24.261 & 724.26 & 317.39 \\
\hline 432.0 & 431.01 & 16.565 & 540.04 & 248.89 & 507.0 & 506.45 & 21.277 & 639.71 & 274.52 & 582.0 & 580.71 & 24.301 & 725.32 & 318.01 \\
\hline 433.0 & 431.95 & 16.638 & 541.44 & 249.26 & 508.0 & 507.46 & 21.318 & 641.79 & 274.88 & 583.0 & 581.69 & 24.342 & 726.37 & 318.63 \\
\hline 434.0 & 432.91 & 16.711 & 542.83 & 249.64 & 509.0 & 508.46 & 21.358 & 643.89 & 275.25 & 584.0 & 582.67 & 24.382 & 727.43 & 319.25 \\
\hline 435.0 & 433.87 & 16.784 & 544.24 & 250.02 & 510.0 & 509.47 & 21.398 & 646.01 & 275.63 & 585.0 & 583.66 & 24.422 & 728.49 & 319.87 \\
\hline 436.0 & 434.84 & 16.857 & 545.64 & 250.41 & 511.0 & 510.47 & 21.438 & 648.10 & 276.00 & 586.0 & 584.65 & 24.462 & 729.56 & 320.49 \\
\hline 437.0 & 435.82 & 16.929 & 547.05 & 250.79 & 512.0 & 511.47 & 21.479 & 650.20 & 276.38 & 587.0 & 585.64 & 24.503 & 730.62 & 321.10 \\
\hline 438.0 & 436.81 & 17.002 & 548.48 & 251.18 & 513.0 & 512.47 & 21.519 & 652.09 & 276.75 & 588.0 & 586.63 & 24.543 & 731.69 & 321.71 \\
\hline 439.0 & 437.81 & 17.075 & 549.89 & 251.56 & 514.0 & 513.47 & 21.559 & 653.61 & 277.14 & 589.0 & 587.63 & 24.583 & 732.77 & 322.32 \\
\hline 440.0 & 438.82 & 17.148 & 551.34 & 251.95 & 515.0 & 514.47 & 21.600 & 655.14 & 277.52 & 590.0 & 588.62 & 24.624 & 733.84 & 322.93 \\
\hline 441.0 & 439.84 & 17.220 & 552.77 & 252.34 & 516.0 & 515.47 & 21.640 & 656.67 & 277.90 & 591.0 & 589.63 & 24.664 & 734.92 & 323.54 \\
\hline
\end{tabular}


Table 2 (continued).

\begin{tabular}{|c|c|c|c|c|c|c|c|c|c|c|c|c|c|c|}
\hline $\begin{array}{r}\text { Hole } \\
807 \mathrm{~A}\end{array}$ & $\begin{array}{l}\text { Hole } \\
807 \mathrm{C}\end{array}$ & Site 807 & $\begin{array}{l}\text { Hole } \\
806 \mathrm{~B}\end{array}$ & $\begin{array}{r}\text { Hole } \\
803 \mathrm{D}\end{array}$ & $\begin{array}{r}\text { Hole } \\
807 \mathrm{~A}\end{array}$ & $\begin{array}{l}\text { Hole } \\
807 \mathrm{C}\end{array}$ & Site 807 & $\begin{array}{l}\text { Hole } \\
806 \mathrm{~B}\end{array}$ & $\begin{array}{c}\text { Hole } \\
\text { 803D }\end{array}$ & $\begin{array}{r}\text { Hole } \\
807 \mathrm{~A}\end{array}$ & $\begin{array}{l}\text { Hole } \\
807 \mathrm{C}\end{array}$ & Site 807 & $\begin{array}{l}\text { Hole } \\
806 \mathrm{~B}\end{array}$ & $\begin{array}{r}\text { Hole } \\
803 \mathrm{D}\end{array}$ \\
\hline $\begin{array}{l}\text { depth } \\
\text { (mbsf) }\end{array}$ & $\begin{array}{l}\text { depth } \\
\text { (mbsf) }\end{array}$ & $\begin{array}{c}\text { age } \\
\text { (Ma) }\end{array}$ & $\begin{array}{c}\text { depth } \\
\text { (mbsf) }\end{array}$ & $\begin{array}{r}\text { depth } \\
\text { (mbsf) }\end{array}$ & $\begin{array}{c}\text { depth } \\
\text { (mbsf) }\end{array}$ & $\begin{array}{l}\text { depth } \\
\text { (mbsf) }\end{array}$ & $\begin{array}{l}\text { age } \\
\text { (Ma) }\end{array}$ & $\begin{array}{l}\text { depth } \\
\text { (mbsf) }\end{array}$ & $\begin{array}{r}\text { depth } \\
\text { (mbsf) }\end{array}$ & $\begin{array}{l}\text { depth } \\
\text { (mbsf) }\end{array}$ & $\begin{array}{c}\text { depth } \\
\text { (mbsf) }\end{array}$ & $\begin{array}{l}\text { age } \\
\text { (Ma) }\end{array}$ & $\begin{array}{c}\text { depth } \\
\text { (mbsf) }\end{array}$ & $\begin{array}{r}\text { depth } \\
\text { (mbsf) }\end{array}$ \\
\hline 592.0 & 590.63 & 24.704 & 736.00 & 324.15 & 667.0 & 667.22 & 27.728 & & 375.80 & 742.0 & 741.69 & 30.752 & & 415.72 \\
\hline 593.0 & 591.63 & 24.745 & 737.07 & 324.76 & 668.0 & 668.18 & 27.769 & & 376.52 & 743.0 & 742.68 & 30.793 & & 416.18 \\
\hline 594.0 & 592.64 & 24.785 & 738.03 & 325.37 & 669.0 & 669.14 & 27.809 & & 377.23 & 744.0 & 743.67 & 30.833 & & 416.65 \\
\hline 595.0 & 593.64 & 24.825 & & 325.97 & 670.0 & 670.10 & 27.849 & & 377.96 & 745.0 & 744.67 & 30.873 & & 417.12 \\
\hline 596.0 & 594.64 & 24.866 & & 326.58 & 671.0 & 671.07 & 27.890 & & 378.68 & 746.0 & 745.67 & 30.914 & & 417.58 \\
\hline 597.0 & 595.64 & 24.906 & & 327.18 & 672.0 & 672.04 & 27.930 & & 379.39 & 747.0 & 746.66 & 30.954 & & 418.05 \\
\hline 598.0 & 596.64 & 24.946 & & 327.79 & 673.0 & 673.02 & 27.970 & & 380.11 & 748.0 & 747.67 & 30.994 & & 418.51 \\
\hline 599.0 & 597.64 & 24.987 & & 328.39 & 674.0 & 674.01 & 28.011 & & 380.83 & 749.0 & 748.67 & 31.035 & & 418.97 \\
\hline 600.0 & 598.64 & 25.027 & & 329.00 & 675.0 & 674.99 & 28.051 & & 381.55 & 750.0 & 749.67 & 31.075 & & 419.43 \\
\hline 601.0 & 599.64 & 25.067 & & 329.60 & 676.0 & 675.99 & 28.091 & & 382.27 & 751.0 & 750.68 & 31.115 & & 419.88 \\
\hline 602.0 & 600.63 & 25.108 & & 330.21 & 677.0 & 676.98 & 28.132 & & 382.99 & 752.0 & 751.68 & 31.156 & & 420.34 \\
\hline 603.0 & 601.62 & 25.148 & & 330.82 & 678.0 & 677.98 & 28.172 & & 383.71 & 753.0 & 752.69 & 31.196 & & 420.79 \\
\hline 604.0 & 602.60 & 25.188 & & 331.42 & 679.0 & 678.99 & 28.212 & & 384.43 & 754.0 & 753.70 & 31.236 & & 421.24 \\
\hline 605.0 & 603.59 & 25.229 & & 332.03 & 680.0 & 680.00 & 28.253 & & 385.16 & 755.0 & 754.70 & 31.277 & & 421.70 \\
\hline 606.0 & 604.57 & 25.269 & & 332.63 & 681.0 & 681.01 & 28.293 & & 385.88 & 756.0 & 755.71 & 31.317 & & 422.15 \\
\hline 607.0 & 605.55 & 25.309 & & 333.24 & 682.0 & 682.03 & 28.333 & & 386.61 & 757.0 & 756.71 & 31.357 & & 422.68 \\
\hline 608.0 & 606.53 & 25.350 & & 333.85 & 683.0 & 683.04 & 28.374 & & 387.33 & 758.0 & 757.72 & 31.398 & & 423.35 \\
\hline 609.0 & 607.50 & 25.390 & & 334.46 & 684.0 & 684.06 & 28.414 & & 388.06 & 759.0 & 758.72 & 31.438 & & 424.22 \\
\hline 610.0 & 608.48 & 25.430 & & 335.08 & 685.0 & 685.07 & 28.454 & & 388.80 & 760.0 & 759.72 & 31.478 & & 425.10 \\
\hline 611.0 & 609.45 & 25.470 & & 335.69 & 686.0 & 686.09 & 28.495 & & 389.32 & 761.0 & 760.72 & 31.519 & & 425.98 \\
\hline 612.0 & 610.43 & 25.511 & & 336.31 & 687.0 & 687.10 & 28.535 & & 389.77 & 762.0 & 761.72 & 31.559 & & 426.85 \\
\hline 613.0 & 611.40 & 25.551 & & 336.92 & 688.0 & 688.11 & 28.575 & & 390.22 & 763.0 & 762.72 & 31.599 & & 427.72 \\
\hline 614.0 & 612.38 & 25.591 & & 337.54 & 689.0 & 689.12 & 28.615 & & 390.67 & 764.0 & 763.71 & 31.639 & & 428.60 \\
\hline 615.0 & 613.35 & 25.632 & & 338.15 & 690.0 & 690.13 & 28.656 & & 391.11 & 765.0 & 764.71 & 31.680 & & 429.47 \\
\hline 616.0 & 614.34 & 25.672 & & 338.77 & 691.0 & 691.13 & 28.696 & & 391.56 & 766.0 & 765.70 & 31.720 & & 430.34 \\
\hline 617.0 & 615.32 & 25.712 & & 339.39 & 692.0 & 692.13 & 28.736 & & 392.01 & 767.0 & 766.70 & 31.760 & & 431.21 \\
\hline 618.0 & 616.30 & 25.753 & & 340.01 & 693.0 & 693.12 & 28.777 & & 392.46 & 768.0 & 767.70 & 31.801 & & 432.08 \\
\hline 619.0 & 617.29 & 25.793 & & 340.63 & 694.0 & 694.11 & 28.817 & & 392.92 & 769.0 & 768.69 & 31.841 & & 432.95 \\
\hline 620.0 & 618.27 & 25.833 & & 341.26 & 695.0 & 695.10 & 28.857 & & 393.37 & 770.0 & 769.68 & 31.881 & & 433.81 \\
\hline 621.0 & 619.25 & 25.874 & & 341.98 & 696.0 & 696.08 & 28.898 & & 393.83 & 771.0 & 770.68 & 31.922 & & 434.68 \\
\hline 622.0 & 620.23 & 25.914 & & 342.72 & 697.0 & 697.07 & 28.938 & & 394.29 & 772.0 & 771.68 & 31.962 & & 435.55 \\
\hline 623.0 & 621.22 & 25.954 & & 343.45 & 698.0 & 698.05 & 28.978 & & 394.74 & 773.0 & 772.67 & 32.002 & & 436.42 \\
\hline 624.0 & 622.21 & 25.995 & & 344.18 & 699.0 & 699.02 & 29.019 & & 395.20 & 774.0 & 773.67 & 32.043 & & 437.29 \\
\hline 625.0 & 623.21 & 26.035 & & 344.92 & 700.0 & 700.00 & 29.059 & & 395.66 & 775.0 & 774.67 & 32.083 & & 438.16 \\
\hline 626.0 & 624.21 & 26.075 & & 345.65 & 701.0 & 700.98 & 29.099 & & 396.12 & 776.0 & 775.67 & 32.123 & & 439.04 \\
\hline 627.0 & 625.22 & 26.116 & & 346.39 & 702.0 & 701.95 & 29.140 & & 396.59 & 777.0 & 776.67 & 32.164 & & 439.92 \\
\hline 628.0 & 626.24 & 26.156 & & 347.13 & 703.0 & 702.93 & 29.180 & & 397.06 & 778.0 & 777.67 & 32.204 & & 440.79 \\
\hline 629.0 & 627.22 & 26.196 & & 347.87 & 704.0 & 703.91 & 29.220 & & 397.52 & 779.0 & 778.67 & 32.244 & & 441.67 \\
\hline 630.0 & 628.19 & 26.237 & & 348.61 & 705.0 & 704.89 & 29.261 & & 397.99 & 780.0 & 779.67 & 32.285 & & 442.55 \\
\hline 631.0 & 629.16 & 26.277 & & 349.35 & 706.0 & 705.87 & 29.301 & & 398.46 & 781.0 & 780.68 & 32.325 & & 443.43 \\
\hline 632.0 & 630.14 & 26.317 & & 350.09 & 707.0 & 706.85 & 29.341 & & 398.93 & 782.0 & 781.68 & 32.365 & & 444.32 \\
\hline 633.0 & 631.12 & 26.358 & & 350.83 & 708.0 & 707.84 & 29.382 & & 399.40 & 783.0 & 782.68 & 32.406 & & 445.20 \\
\hline 634.0 & 632.11 & 26.398 & & 351.57 & 709.0 & 708.83 & 29.422 & & 399.88 & 784.0 & 783.69 & 32.446 & & 446.10 \\
\hline 635.0 & 633.10 & 26.438 & & 352.32 & 710.0 & 709.83 & 29.462 & & 400.36 & 785.0 & 784.69 & 32.486 & & 446.99 \\
\hline 636.0 & 634.09 & 26.479 & & 353.06 & 711.0 & 710.82 & 29.503 & & 400.83 & 786.0 & 785.69 & 32.527 & & 447.89 \\
\hline 637.0 & 635.09 & 26.519 & & 353.80 & 712.0 & 711.82 & 29.543 & & 401.31 & 787.0 & 786.69 & 32.567 & & 448.79 \\
\hline 638.0 & 636.09 & 26.559 & & 354.54 & 713.0 & 712.82 & 29.583 & & 401.79 & 788.0 & 787.69 & 32.607 & & 449.70 \\
\hline 639.0 & 637.10 & 26.599 & & 355.29 & 714.0 & 713.83 & 29.623 & & 402.27 & 789.0 & 788.69 & 32.647 & & 450.61 \\
\hline 640.0 & 638.12 & 26.640 & & 356.03 & 715.0 & 714.84 & 29.664 & & 402.75 & 790.0 & 789.69 & 32.688 & & 451.52 \\
\hline 641.0 & 639.13 & 26.680 & & 356.77 & 716.0 & 715.84 & 29.704 & & 403.23 & 791.0 & 790.69 & 32.728 & & 452.44 \\
\hline 642.0 & 640.14 & 26.720 & & 357.52 & 717.0 & 716.85 & 29.744 & & 403.71 & 792.0 & 791.68 & 32.768 & & 453.35 \\
\hline 643.0 & 641.15 & 26.761 & & 358.26 & 718.0 & 717.86 & 29.785 & & 404.19 & 793.0 & 792.68 & 32.809 & & 454.27 \\
\hline 644.0 & 642.16 & 26.801 & & 359.00 & 719.0 & 718.87 & 29.825 & & 404.68 & 794.0 & 793.67 & 32.849 & & 455.19 \\
\hline 645.0 & 643.16 & 26.841 & & 359.73 & 720.0 & 719.88 & 29.865 & & 405.16 & 795.0 & 794.67 & 32.889 & & 456.12 \\
\hline 646.0 & 644.16 & 26.882 & & 360.48 & 721.0 & 720.89 & 29.906 & & 405.64 & 796.0 & 795.66 & 32.930 & & 457.05 \\
\hline 647.0 & 645.34 & 26.922 & & 361.22 & 722.0 & 721.89 & 29.946 & & 406.13 & 797.0 & 796.66 & 32.970 & & 457.99 \\
\hline 648.0 & 646.56 & 26.962 & & 361.96 & 723.0 & 722.90 & 29.986 & & 406.62 & 798.0 & 797.65 & 33.010 & & 458.93 \\
\hline 649.0 & 647.77 & 27.003 & & 362.69 & 724.0 & 723.90 & 30.027 & & 407.10 & 799.0 & 798.65 & 33.051 & & 459.86 \\
\hline 650.0 & 648.96 & 27.043 & & 363.43 & 725.0 & 724.90 & 30.067 & & 407.58 & 800.0 & 799.64 & 33.091 & & 460.81 \\
\hline 651.0 & 650.15 & 27.083 & & 364.17 & 726.0 & 725.90 & 30.107 & & 408.07 & 801.0 & 800.64 & 33.131 & & 461.75 \\
\hline 652.0 & 651.33 & 27.124 & & 364.90 & 727.0 & 726.89 & 30.148 & & 408.55 & 802.0 & 801.64 & 33.172 & & 462.69 \\
\hline 653.0 & 652.50 & 27.164 & & 365.63 & 728.0 & 727.89 & 30.188 & & 409.03 & 803.0 & 802.64 & 33.212 & & 463.64 \\
\hline 654.0 & 653.66 & 27.204 & & 366.37 & 729.0 & 728.88 & 30.228 & & 409.52 & 804.0 & 803.65 & 33.252 & & 464.58 \\
\hline 655.0 & 654.81 & 27.245 & & 367.10 & 730.0 & 729.87 & 30.269 & & 410.00 & 805.0 & 804.65 & 33.293 & & 465.54 \\
\hline 656.0 & 655.95 & 27.285 & & 367.83 & 731.0 & 730.85 & 30.309 & & 410.48 & 806.0 & 805.66 & 33.333 & & 466.49 \\
\hline 657.0 & 657.09 & 27.325 & & 368.56 & 732.0 & 731.84 & 30.349 & & 410.96 & 807.0 & 806.68 & 33.373 & & 467.43 \\
\hline 658.0 & 658.22 & 27.366 & & 369.29 & 733.0 & 732.82 & 30.390 & & 411.44 & 808.0 & 807.70 & 33.414 & & 468.38 \\
\hline 659.0 & 659.34 & 27.406 & & 370.02 & 734.0 & 733.81 & 30.430 & & 411.92 & 809.0 & 808.72 & 33.454 & & 469.34 \\
\hline 660.0 & 660.46 & 27.446 & & 370.74 & 735.0 & 734.79 & 30.470 & & 412.40 & 810.0 & 809.74 & 33.494 & & 470.29 \\
\hline 661.0 & 661.47 & 27.487 & & 371.47 & 736.0 & 735.77 & 30.511 & & 412.88 & 811.0 & 810.77 & 33.535 & & 471.23 \\
\hline 662.0 & 662.43 & 27.527 & & 372.19 & 737.0 & 736.76 & 30.551 & & 413.35 & 812.0 & 811.80 & 33.575 & & 472.18 \\
\hline 663.0 & 663.39 & 27.567 & & 372.91 & 738.0 & 737.74 & 30.591 & & 413.83 & 813.0 & 812.84 & 33.615 & & 473.13 \\
\hline 664.0 & 664.35 & 27.607 & & 373.64 & 739.0 & 738.73 & 30.631 & & 414.30 & 814.0 & 813.88 & 33.655 & & 474.07 \\
\hline 665.0 & 665.30 & 27.648 & & 374.36 & 740.0 & 739.71 & 30.672 & & 414.78 & 815.0 & 814.92 & 33.696 & & 475.01 \\
\hline 666.0 & 666.26 & 27.688 & & 375.08 & 741.0 & 740.70 & 30.712 & & 415.25 & 816.0 & 815.96 & 33.736 & & 475.95 \\
\hline
\end{tabular}


Table 2 (continued),

\begin{tabular}{|c|c|c|c|c|c|c|c|c|c|c|c|c|c|c|}
\hline $\begin{array}{l}\text { Hole } \\
807 \mathrm{~A}\end{array}$ & $\begin{array}{l}\text { Hole } \\
807 \mathrm{C}\end{array}$ & & $\begin{array}{l}\text { Hole } \\
806 \mathrm{~B}\end{array}$ & $\begin{array}{l}\text { Hole } \\
803 \mathrm{D}\end{array}$ & $\begin{array}{l}\text { Hole } \\
807 \mathrm{~A}\end{array}$ & $\begin{array}{l}\text { Hole } \\
807 \mathrm{C}\end{array}$ & Site 807 & $\begin{array}{l}\text { Hole } \\
806 \mathrm{~B}\end{array}$ & $\begin{array}{l}\text { Hole } \\
803 \mathrm{D}\end{array}$ & $\begin{array}{l}\text { Hole } \\
807 \mathrm{~A}\end{array}$ & Hole & Site 807 & $\begin{array}{l}\text { Hole } \\
806 \mathrm{~B}\end{array}$ & Hole \\
\hline $\begin{array}{l}\text { depth } \\
\text { (mbsf) }\end{array}$ & $\begin{array}{l}\text { depth } \\
\text { (mbsf) }\end{array}$ & $\begin{array}{l}\text { age } \\
\text { (Ma) }\end{array}$ & $\begin{array}{l}\text { depth } \\
\text { (mbsf) }\end{array}$ & $\begin{array}{r}\text { depth } \\
\text { (mbsf) }\end{array}$ & $\begin{array}{l}\text { depth } \\
\text { (mbsf) }\end{array}$ & $\begin{array}{l}\text { depth } \\
\text { (mbsf) }\end{array}$ & $\begin{array}{c}\text { age } \\
\text { (Ma) }\end{array}$ & $\begin{array}{l}\text { depth } \\
\text { (mbsf) }\end{array}$ & $\begin{array}{l}\text { depth } \\
\text { (mbsf) }\end{array}$ & $\begin{array}{c}\text { depth } \\
\text { (mbsf) }\end{array}$ & $\begin{array}{l}\text { depth } \\
\text { (mbsf) }\end{array}$ & $\begin{array}{c}\text { age } \\
(\mathrm{Ma})\end{array}$ & $\begin{array}{l}\text { depth } \\
\text { (mbsf) }\end{array}$ & $\begin{array}{r}\text { depth } \\
\text { (mbsf) }\end{array}$ \\
\hline 817.0 & 817.00 & 33.776 & & 476.88 & & 879.00 & 36.276 & & 523.41 & & 941.00 & 38.776 & & 557.93 \\
\hline \multirow[t]{61}{*}{818.0} & 818.05 & 33.817 & & 477.81 & & 880.00 & 36.317 & & 524.01 & & 942.00 & 38.816 & & 558.46 \\
\hline & 819.00 & 33.857 & & 478.73 & & 881.00 & 36.357 & & 524.60 & & 943.00 & 38.857 & & 558.99 \\
\hline & 820.00 & 33.897 & & 479.66 & & 882.00 & 36.397 & & 525.20 & & 944.00 & 38.897 & & 559.53 \\
\hline & 821.00 & 33.938 & & 480.58 & & 883.00 & 36.438 & & 525.80 & & 945.00 & 38.937 & & 560.07 \\
\hline & 822.00 & 33.978 & & 481.49 & & 884.00 & 36.478 & & 526.40 & & 946.00 & 38.978 & & 560.60 \\
\hline & 823.00 & 34.018 & & 482.39 & & 885.00 & 36.518 & & 527.01 & & 947.00 & 39.018 & & 561.14 \\
\hline & 824.00 & 34.059 & & 483.30 & & 886.00 & 36.559 & & 527.61 & & 948.00 & 39.058 & & 561.68 \\
\hline & 825.00 & 34.099 & & 484.19 & & 887.00 & 36.599 & & 528.22 & & 949.00 & 39.099 & & 562.23 \\
\hline & 826.00 & 34.139 & & 485.08 & & 888.00 & 36.639 & & 528.83 & & 950.00 & 39.139 & & 562.77 \\
\hline & 827.00 & 34.180 & & 485.96 & & 889.00 & 36.680 & & 529.44 & & 951.00 & 39.179 & & 563.32 \\
\hline & 828.00 & 34.220 & & 486.85 & & 890.00 & 36.720 & & 530.06 & & 952.00 & 39.220 & & 563.87 \\
\hline & 829.00 & 34.260 & & 487.72 & & 891.00 & 36.760 & & 530.68 & & 953.00 & 39.260 & & 564.42 \\
\hline & 830.00 & 34.301 & & 488.58 & & 892.00 & 36.800 & & 531.30 & & 954.00 & 39.300 & & 564.98 \\
\hline & 831.00 & 34.341 & & 489.43 & & 893.00 & 36.841 & & 531.92 & & 955.00 & 39.341 & & 565.53 \\
\hline & 832.00 & 34.381 & & 490.28 & & 894.00 & 36.881 & & 532.55 & & 956.00 & 39.381 & & 566.09 \\
\hline & 833.00 & 34.422 & & 491.13 & & 895.00 & 36.921 & & 533.18 & & 957.00 & 39.421 & & 566.66 \\
\hline & 834.00 & 34.462 & & 491.97 & & 896.00 & 36.962 & & 533.82 & & 958.00 & 39.462 & & 567.23 \\
\hline & 835.00 & 34.502 & & 492.79 & & 897.00 & 37.002 & & 534.45 & & 959.00 & 39.502 & & 567.80 \\
\hline & 836.00 & 34.543 & & 493.62 & & 898.00 & 37.042 & & 535.09 & & 960.00 & 39.542 & & 568.37 \\
\hline & 837.00 & 34.583 & & 494.44 & & 899.00 & 37.083 & & 535.73 & & 961.00 & 39.583 & & 568.95 \\
\hline & 838.00 & 34.623 & & 495.24 & & 900.00 & 37.123 & & 536.37 & & 962.00 & 39.623 & & 569.54 \\
\hline & 839.00 & 34.663 & & 496.04 & & 901.00 & 37.163 & & 537.02 & & 963.00 & 39.663 & & 570.12 \\
\hline & 840.00 & 34.704 & & 496.83 & & 902.00 & 37.204 & & 537.61 & & 964.00 & 39.704 & & 570.72 \\
\hline & 841.00 & 34.744 & & 497.63 & & 903.00 & 37.244 & & 538.09 & & 965.00 & 39.744 & & 571.32 \\
\hline & 842.00 & 34.784 & & 498.41 & & 904.00 & 37.284 & & 538.59 & & 966.00 & 39.784 & & 571.91 \\
\hline & 843.00 & 34.825 & & 499.18 & & 905.00 & 37.325 & & 539.09 & & 967.00 & 39.824 & & 572.52 \\
\hline & 844.00 & 34.865 & & 499.96 & & 906.00 & 37.365 & & 539.60 & & 968.00 & 39.865 & & 573.13 \\
\hline & 845.00 & 34.905 & & 500.77 & & 907.00 & 37.405 & & 540.10 & & 969.00 & 39.905 & & 573.75 \\
\hline & 846.00 & 34.946 & & 501.56 & & 908.00 & 37.446 & & 540.61 & & 970.00 & 39.945 & & 574.38 \\
\hline & 847.00 & 34.986 & & 502.36 & & 909.00 & 37.486 & & 541.13 & & 971.00 & 39.986 & & 575.00 \\
\hline & 848.00 & 35.026 & & 503.15 & & 910.00 & 37.526 & & 541.64 & & 972.00 & 40.026 & & 575.64 \\
\hline & 849.00 & 35.067 & & 503.93 & & 911.00 & 37.567 & & 542.15 & & 973.00 & 40.066 & & 576.27 \\
\hline & 850.00 & 35.107 & & 504.71 & & 912.00 & 37.607 & & 542.67 & & 974.00 & 40.107 & & 576.91 \\
\hline & 851.00 & 35.147 & & 505.48 & & 913.00 & 37.647 & & 543.19 & & 975.00 & 40.147 & & 577.57 \\
\hline & 852.00 & 35.188 & & 506.24 & & 914.00 & 37.688 & & 543.70 & & 976.00 & 40.187 & & 578.22 \\
\hline & 853.00 & 35.228 & & 507.00 & & 915.00 & 37.728 & & 544.22 & & 977.00 & 40.228 & & 578.88 \\
\hline & 854.00 & 35.268 & & 507.76 & & 916.00 & 37.768 & & 544.74 & & 978.00 & 40.268 & & 579.54 \\
\hline & 855.00 & 35.309 & & 508.51 & & 917.00 & 37.808 & & 545.27 & & 979.00 & 40.308 & & 580.21 \\
\hline & 856.00 & 35.349 & & 509.26 & & 918.00 & 37.849 & & 545.79 & & 980.00 & 40.349 & & 580.89 \\
\hline & 857.00 & 35.389 & & 510.00 & & 919.00 & 37.889 & & 546.31 & & 981.00 & 40.389 & & 581.56 \\
\hline & 858.00 & 35.430 & & 510.73 & & 920.00 & 37.929 & & 546.84 & & 982.00 & 40.429 & & 582.25 \\
\hline & 859.00 & 35.470 & & 511.47 & & 921.00 & 37.970 & & 547.36 & & 983.00 & 40.470 & & 582.94 \\
\hline & 860.00 & 35.510 & & 512.16 & & 922.00 & 38.010 & & 547.89 & & 984.00 & 40.510 & & 583.63 \\
\hline & 861.00 & 35.551 & & 512.76 & & 923.00 & 38.050 & & 548.41 & & 985.00 & 40.550 & & 584.33 \\
\hline & 862.00 & 35.591 & & 513.36 & & 924.00 & 38.091 & & 548.94 & & 986.00 & 40.591 & & 585.04 \\
\hline & 863.00 & 35.631 & & 513.95 & & 925.00 & 38.131 & & 549.47 & & 987.00 & 40.631 & & 585.74 \\
\hline & 864.00 & 35.671 & & 514.55 & & 926.00 & 38.171 & & 549.99 & & 988.00 & 40.671 & & 586.46 \\
\hline & 865.00 & 35.712 & & 515.14 & & 927.00 & 38.212 & & 550.52 & & 989.00 & 40.712 & & 587.17 \\
\hline & 866.00 & 35.752 & & 515.74 & & 928.00 & 38.252 & & 551.05 & & 990.00 & 40.752 & & 587.89 \\
\hline & 867.00 & 35.792 & & 516.33 & & 929.00 & 38.292 & & 551.57 & & 991.00 & 40.792 & & 588.78 \\
\hline & 868.00 & 35.833 & & 516.92 & & 930.00 & 38.333 & & 552.10 & & 992.00 & 40.832 & & 589.86 \\
\hline & 869.00 & 35.873 & & 517.51 & & 931.00 & 38.373 & & 552.63 & & 993.00 & 40.873 & & 590.94 \\
\hline & 870.00 & 35.913 & & 518.10 & & 932.00 & 38.413 & & 553.16 & & 994.00 & 40.913 & & 592.01 \\
\hline & 871.00 & 35.954 & & 518.69 & & 933.00 & 38.454 & & 553.69 & & 995.00 & 40.953 & & 593.10 \\
\hline & 872.00 & 35.994 & & 519.28 & & 934.00 & 38.494 & & 554.22 & & 996.00 & 40.994 & & 594.18 \\
\hline & 873.00 & 36.034 & & 519.87 & & 935.00 & 38.534 & & 554.74 & & 997.00 & 41.034 & & 595.45 \\
\hline & 874,00 & 36.075 & & 520.45 & & 936.00 & 38.575 & & 555.28 & & 998.00 & 41.074 & & 596.74 \\
\hline & 875.00 & 36.115 & & 521.05 & & 937.00 & 38.615 & & 555.80 & & 999.00 & 41.115 & & 598.03 \\
\hline & 876.00 & 36.155 & & 521.64 & & 938.00 & 38.655 & & 556.34 & & 1000.00 & 41.155 & & 599.33 \\
\hline & 877.00 & 36.196 & & 522.23 & & 939.00 & 38.696 & & 556.86 & & & & & \\
\hline & 878.00 & 36.236 & & 522.82 & & 940.00 & 38.736 & & 557.39 & & & & & \\
\hline
\end{tabular}

Note: Equivalent depths are based upon the well-log correlation between Holes 807A and 807C and Holes 806B and 803D. Age scale is from Site 807 and is preliminary. 


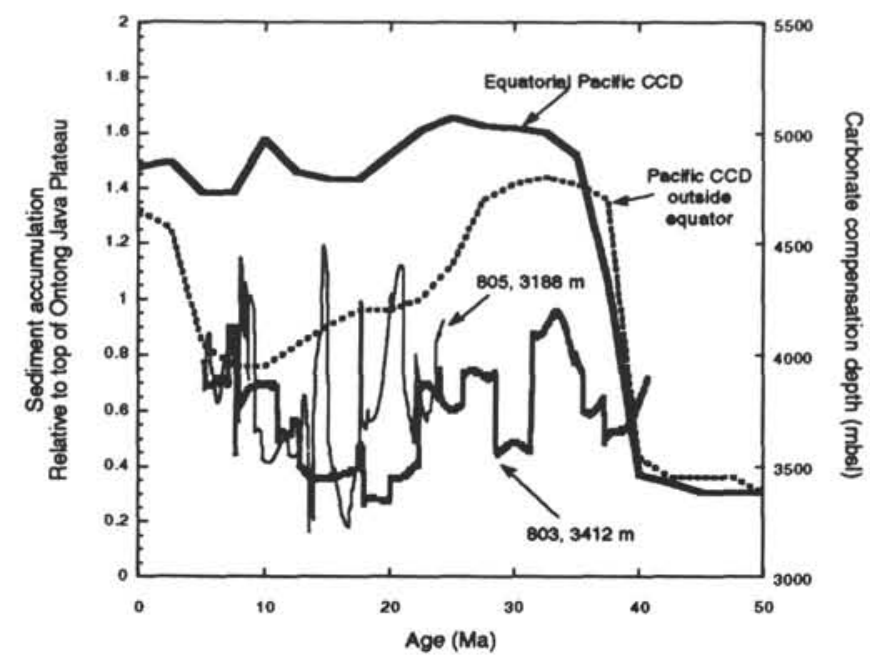

Figure 15. Sediment accumulation down the flank of the Ontong Java Plateau relative to drill sites on the top of the plateau. Also shown are previous estimates of the Pacific CCD from DSDP drilling (van Andel and Moore, 1974; van Andel et al., 1975). The CCD data were inverted so that sediment accumulation and $\mathrm{CCD}$ changes should have the same sense of direction. The deepest Ontong Java Plateau site logged, Hole 803D, shows an accumulation pattern vaguely resembling the equatorial $\mathrm{CCD}$, but the intermediate site, Hole 805 , alternates between accumulating sediments at the same rate as the top of the plateau and Hole 803D.

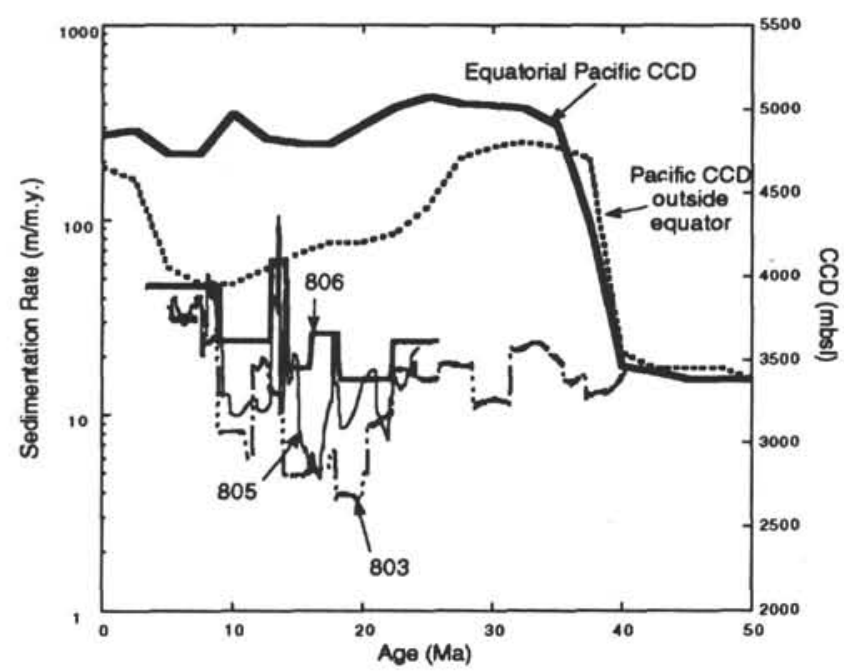

Figure 16. Sedimentation rates of three Ontong Java Plateau sites that form a transect down the flank of the plateau. Sedimentation rates, a crude measure of carbonate accumulation, show no resemblance to the earlier CCD information. Sedimentation on the flanks of the Ontong Java Plateau does not appear to have been controlled by depth-dependent carbonate dissolution. 